\title{
New insights on unspecific peroxygenases: superfamily reclassification and evolution
}

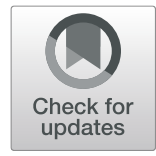

Muniba Faiza', Shengfeng Huang ${ }^{2,3^{*}}$, Dongming $\operatorname{Lan}^{1}$ and Yonghua Wang ${ }^{1 *}$

\begin{abstract}
Background: Unspecific peroxygenases (UPO) (EC 1.11.2.1) represent an intriguing oxidoreductase sub-subclass of heme proteins with peroxygenase and peroxidase activity. With over 300 identified substrates, UPOs catalyze numerous oxidations including 1- or 2- electron oxygenation, selective oxyfunctionalizations, which make them most significant in organic syntheses and potentially attractive as industrial biocatalysts. There are very few UPOs available with distinct properties, notably, MroUPO which shows behavior ranging between UPO and another heme-thiolate peroxidase, called Chloroperoxidase (CPO). It prompted us to search for more UPOs in fungal kingdom which led us to studying their relationship with CPO.

Results: In this study, we searched for novel UPOs in more than 800 fungal genomes and found 113 putative UPOencoding sequences distributed in 35 different fungal species (or strains), amongst which single sequence per species were subjected to phylogeny study along with CPOs. Our phylogenetic study show that the UPOs are distributed in Basidiomycota and Ascomycota phyla of fungi. The sequence analysis helped to classify the UPOs into five distinct subfamilies: classic AaeUPO and four new subfamilies with potential new traits. We have also shown that each of these five subfamilies (supported by) have their own signature motifs. Surprisingly, some of the CPOs appeared to be a type of UPOs indicating that they were previously identified incorrectly. Selection pressure was observed on important motifs in UPOs which could have driven their functional divergence. Furthermore, the sites having different evolutionary rates caused by the functional divergence were also identified on some motifs along with the other relevant amino acid residues. Finally, we predicted critical amino acids responsible for the functional divergence in the UPOs and identified some sequence differences among UPOs, CPOs, and MroUPO to predict it's ranging behavior.

Conclusion: This study discovers new UPOs, provides a glimpse of their evolution from CPOs, and presents new insight on their functional divergence. We present a new classification of UPOs and shed new light on its phylogenetics. These different UPOs may exhibit a wide range of characteristics and specificities which may help in various fields of synthetic chemistry and industrial biocatalysts, and may as well lead to an advancement towards the understanding of physiological role of UPOs in fungi.
\end{abstract}

Keywords: Fungal genomes, Unspecific peroxygenases, Agrocybe aegerita, Chloroperoxidase, Phylogeny, Selection pressure, Functional divergence, Subfamilies, Superfamily

\footnotetext{
* Correspondence: hshengf2@mail.sysu.edu.cn; yonghw@scut.edu.cn

²aboratory for Marine Biology and Biotechnology, Qingdao National

Laboratory for Marine Science and Technology, Qingdao, China

${ }^{1}$ School of Food Science and Engineering, South China University of

Technology, Guangzhou 510640, China

Full list of author information is available at the end of the article
}

(c) The Author(s). 2019 Open Access This article is distributed under the terms of the Creative Commons Attribution 4.0 International License (http://creativecommons.org/licenses/by/4.0/), which permits unrestricted use, distribution, and reproduction in any medium, provided you give appropriate credit to the original author(s) and the source, provide a link to the Creative Commons license, and indicate if changes were made. The Creative Commons Public Domain Dedication waiver (http://creativecommons.org/publicdomain/zero/1.0/) applies to the data made available in this article, unless otherwise stated. 


\section{Introduction}

Unspecific peroxygenase (UPO), also known as aromatic peroxygenase (APO), are newly discovered extracellular enzymes which belong to heme-thiolate proteins obtained from fungal species [1]. The first UPO enzyme was discovered in Agrocybe aegerita (AaeUPO) which belongs to Basidiomycota, commonly known as Black Poplar mushroom [2,3]. AaeUPO is known to catalyze a number of reactions leading to the formation of alcohol by transfer of an oxygen atom by reacting with hydrogen peroxide [4-6]. Fungal UPOs are characterized for catalyzing a large variety of reactions such as epoxidation, hydroxylation, dealkylations, oxidation of aromatic and heterocyclic compounds, organic heteroatoms, inorganic halides, and one- and two- electron oxidations as well [6-8]. They exhibit various useful properties such as high specific activity, catalytic activity, and specificity, catalyze reactions with inexpensive peroxides and cofactors $\left(\mathrm{Mg}^{2+}\right)$, stability and it is water-soluble in nature due to a high degree of glycosylation. Hence, they are considered very intriguing enzymes and also termed as 'closest to ideal biocatalysts for (sub)-terminal hydroxylation of short-chain and medium-chain alkanes under mild conditions' [9]. The other known fungal UPOs include Marasmius rotula (MroUPO) and Coprinellus radians (CraUPO). Like AaeUPO, MroUPO and CraUPO also belong to order Agaricales (known as "gilled mushrooms") of Basidiomycota phylum.

Chloroperoxidase (CPO) (EC 1.11.1.10) is a well-known heme-thiolate peroxidase (HTP), which has strong peroxidase activity but unlike UPOs, it falls short on peroxygenating aromatic substrates and strong $\mathrm{C}-\mathrm{H}$ bonds [10]. UPOs are classified as HTPs due to their characteristic heme ligation by a cysteine and resemblance to CPOs. UPOs are generally present in Dikarya, higher fungi that includes the two phylum: Ascomycota and Basidiomycota of the fungal kingdom as also supported by the results obtained in this study, and found lacking in Taphrinomycotina, Saccharomycotina involving true yeasts such as Saccharomyces, fission yeasts such as
Schizosaccharomyces, all other fungal species as also reported earlier [11, 12]. Based on their molecular mass and motif patterns, UPOs are classified into two categories: Group-I (short UPO sequences) with an average mass of $29 \mathrm{kDa}$ and Group-II (long UPO sequences) with an average mass of $44 \mathrm{kDa}$ [13]. MroUPO (and interestingly, CPO) belong to Group-I UPOs which are widespread in the whole fungal phyla whereas AaeUPO and CraUPO belong to Group-II UPOs which are found only in Ascomycota and Basidiomycota.

To date, only two peroxygenase protein crystal structures are available in Protein Data Bank (PDB): Agrocybe aegerita (PDB ID: 2YOR) and Marasmius rotula (PDB ID: 5FUJ/5FUK), of which the structure of AaeUPO solved at $2.2 \AA$ [14] is well studied (Fig. 1). Although as compared to AaeUPO, MroUPO shows less peroxygenating activity [15] but it is capable of oxidizing bulkier substrates [16] and produces higher protein yields [15]. The distinctive feature of MroUPO is its limited capability of oxidizing iodide amongst the other halides and hence lacking brominating or chlorinating activities. Although its catalytic behavior towards the peroxidase substrates such as dimethoxyphenol, and peroxygenation of aryl alcohols is similar to the AaeUPO and a chloroperoxidase (CPO) namely, Leptoxyphium fumago CPO (LfuCPO); and the oxygen transfer potential is slightly higher than that of $L f u C P O[1,17,18]$. Another most relevant catalytic property of MroUPO is its ability to transfer peroxide-borne oxygen to non-activated carbon, on the basis of which its behavior was stated ranging between AaeUPO and LfuCPO [15]. These different properties of MroUPO ranging between AaeUPO and LfuCPO, suggest its link between both these enzymes. Therefore, we hypothesized that the MroUPO may be bridging a gap between CPOs and UPOs, which despite having motifs similar to CPOs, is capable of functioning as peroxygenase as well. To analyze the relationship between these UPOs and CPOs, we searched for new UPOs in the fungal kingdom.

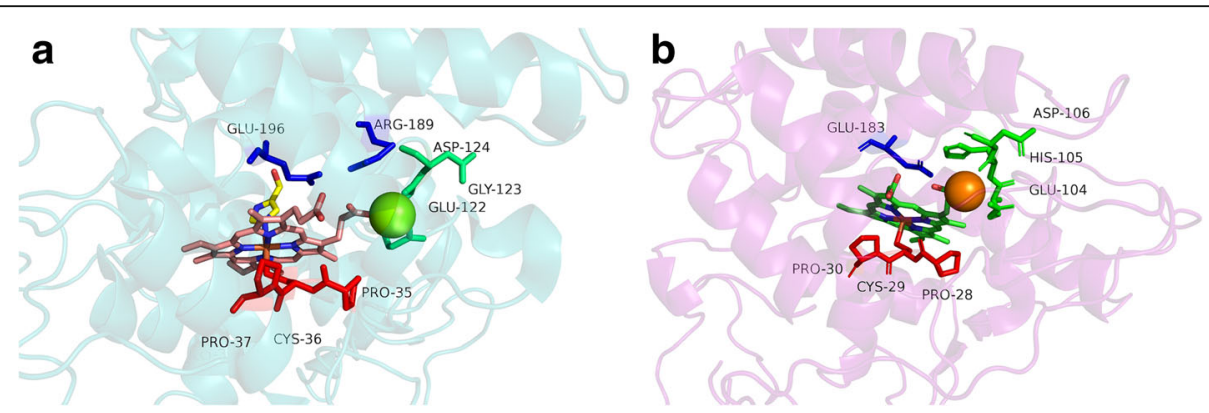

Fig. 1 represents the experimentally resolved structures showing conserved motifs in a) AaeUPO b) LfuCPO. The PCP motifs are shown with red sticks, EGD and EHD motifs are shown in green, acid-base catalyst in $\mathbf{a}$ ) and charge stabilizer in $\mathbf{b}$ ) are shown in blue 
In order to correctly identify the UPO encoding sequences in fungal genomes, the structural properties and catalytic motifs were thoroughly analyzed in both AaeUPO and LfuCPO. The AaeUPO has a cone-shaped cavity constituting a binding pocket for substrates, which is surrounded by aromatic residues [14]. Since both UPO and CPO belong to the HTP superfamily, they both have the same PCP (Proline-Cysteine-Proline) motif with the Cys36 (in AaeUPO) and Cys29 (in $L f u C P O)$ being the proximal ligand for the heme (Fig. 1). This motif is highly conserved in UPOs and required for their catalytic activity [14]. In both the enzymes, the distal heme cavity consists of a negatively charged residue Glu196 in AaeUPO and Glu183 in CPO. The charge stabilizer in AaeUPO is Arg189 and that in CPO is His105 [14]. Arg189-Glu196 acts as the acid-base catalyst pair in AaeUPO while in CPO this function is performed by His105-Glu183. This acid-base catalyst pair is crucial for the formation of Compound-I in all peroxidases $[19,20]$ including both CPOs and UPOs during their catalytic process. The His 105 residue is involved in peroxidase function of $\mathrm{CPO}$ by participating indirectly in the cleavage of the peroxide bond by forming a hydrogen-bond to direct Glu183 in the heme center [21]. The third motif required for the catalytic properties of AaeUPO is EGD motif (i.e., Glu122-Gly123-Asp124), which is slightly different in CPO where Gly123 present in AaeUPO is replaced by His in CPO forming EHD motif $[14,22]$. Previously, it was also proposed that the His residue of EHD motif in CPO is exchanged by the Gly123 in AaeUPO due to which they both do not follow the same mechanism of catalysis [22]. However, there is no strong evidence to support this hypothesis. Since there are not many structural details available about MroUPO, therefore, the motifs were analyzed manually in the sequence of MroUPO revealing the same pattern as exhibited by the CPOs. To summarize, conserved motif patterns for the catalytic activity of AaeUPO is -PCP-EGD-R--E, and for MroUPO and CPO is -PCP-EHD-E [7, 22].

Both UPO and CPO are HTPs, both have to undergo the formation of Compound-I in their catalytic processes, and have structural similarities but show different catalytic properties such as CPO is a typical haloperoxidase while UPO displays relatively weaker haloperoxidase activity and predominates at showing peroxygenase property [7]. Therefore, we have used fungal CPOs as the outgroup for the comparison. Since MroUPO exhibits the same motif pattern as possessed by the CPOs, therefore, it was placed among CPOs for further analyses. In this study, we have customized a genome data mining pipeline to search for novel UPOs in 812 fungal genomes available in the Ensembl database.

\section{Results}

Novel UPO encoding putative sequences in the fungal kingdom

It has been stated that there are thousands of UPO-like sequences present in databases [7] but perhaps these sequences may lack the catalytic motifs. Therefore, both homology search and motif search helped to eliminate the extra sequences which are lacking the required motifs for the catalytic activity of UPO. Finally, we obtained 113 putative UPO sequences from the fungal kingdom which belong to 35 different fungal species including different strains. The largest number of putative sequences come from Sphaerobolus stellatus ss14 (26 putative sequences) followed by Galerina marginata cbs339.88 (11 putative sequences), Agaricus bisporus var burnettii jb137s8 (10 putative sequences), Exidia glandulosa hhb12029 (9 putative sequences), Sistotremastrum niveocremeum hhb9708 and Hypholoma sublaterium fd334ss4 (6 putative sequences), Coprinopsis cinerea okayama7.130 (5 putative sequences), Fibulorhizoctonia sp cbs109695 (4 putative sequences), and rest of the species consists of 1-3 putative sequences as shown in (Additional file 1: Table S1). The largest number of putative sequences are gilled mushrooms and come from Agaricomycotina subphylum of Basidiomycota showing high similarity to AaeUPO. It shows that most of the putative fungal UPOs reside in Basidiomycota phylum of fungal kingdom.

\section{Phylogenetic analysis}

The LBA analysis of the constructed phylogeny revealed that the sequences are evolving along a completely resolved phylogeny (Fig. 2) [23]. The proportion of points inside the matrix increases with the length of sequences indicating that the noise caused by sampling artifacts is diminished. The estimated branch lengths of the constructed ML tree were significantly lower showing less amount of change along the sequences (Fig. 3).

The constructed phylogenetic tree of UPO and CPO sequences showed that most of the obtained UPOs belong to the Basidiomycota phylum distributed in three subphyla: Agaricomycotina, Pucciniomycotina, and Ustilaginomycotina. The majority of UPOs belong to Agaricomycotina as it constitutes around $70 \%$ of the Basidiomycota followed by Ustilaginomycotina and Pucciniomycotina. Only Pezizomycotina subphylum of Ascomycota was found to be consists of UPO, and found lacking in Wallemiomycotina subphylum of Basidiomycota, and Taphrinomycotina and Saccharomycotina subphyla of Ascomycota. Besides, there was only one fungal genus which consists of both UPO as well as CPO, namely, Agaricus which consists of CPO in Agaricus bisporus and Agaricus bisporus var bisporus $\mathrm{H97}$ whereas UPO in Agaricus bisporus 


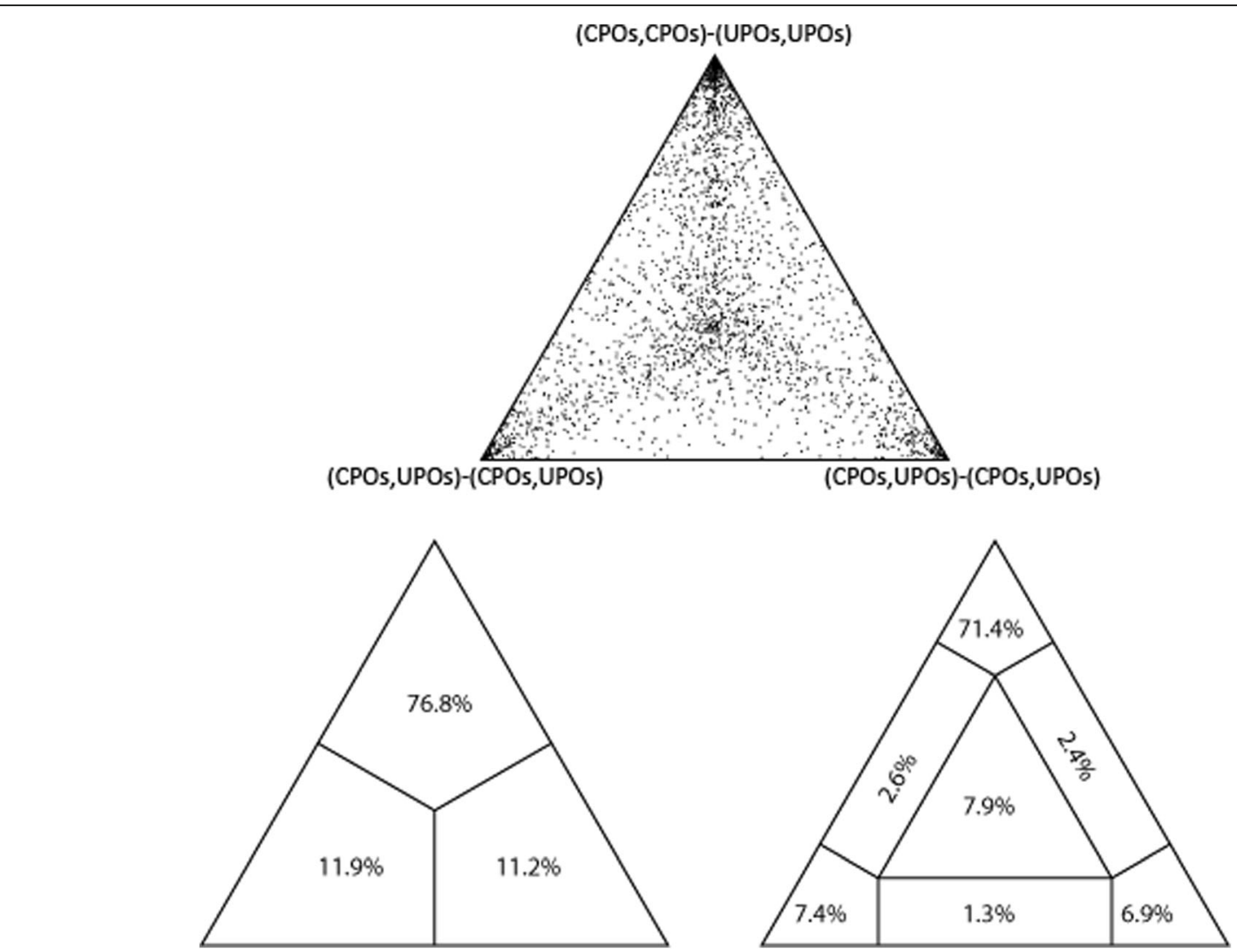

Fig. 2 LBA of the constructed phylogeny of CPOs and resultant UPOs showing the completely resolved phylogeny

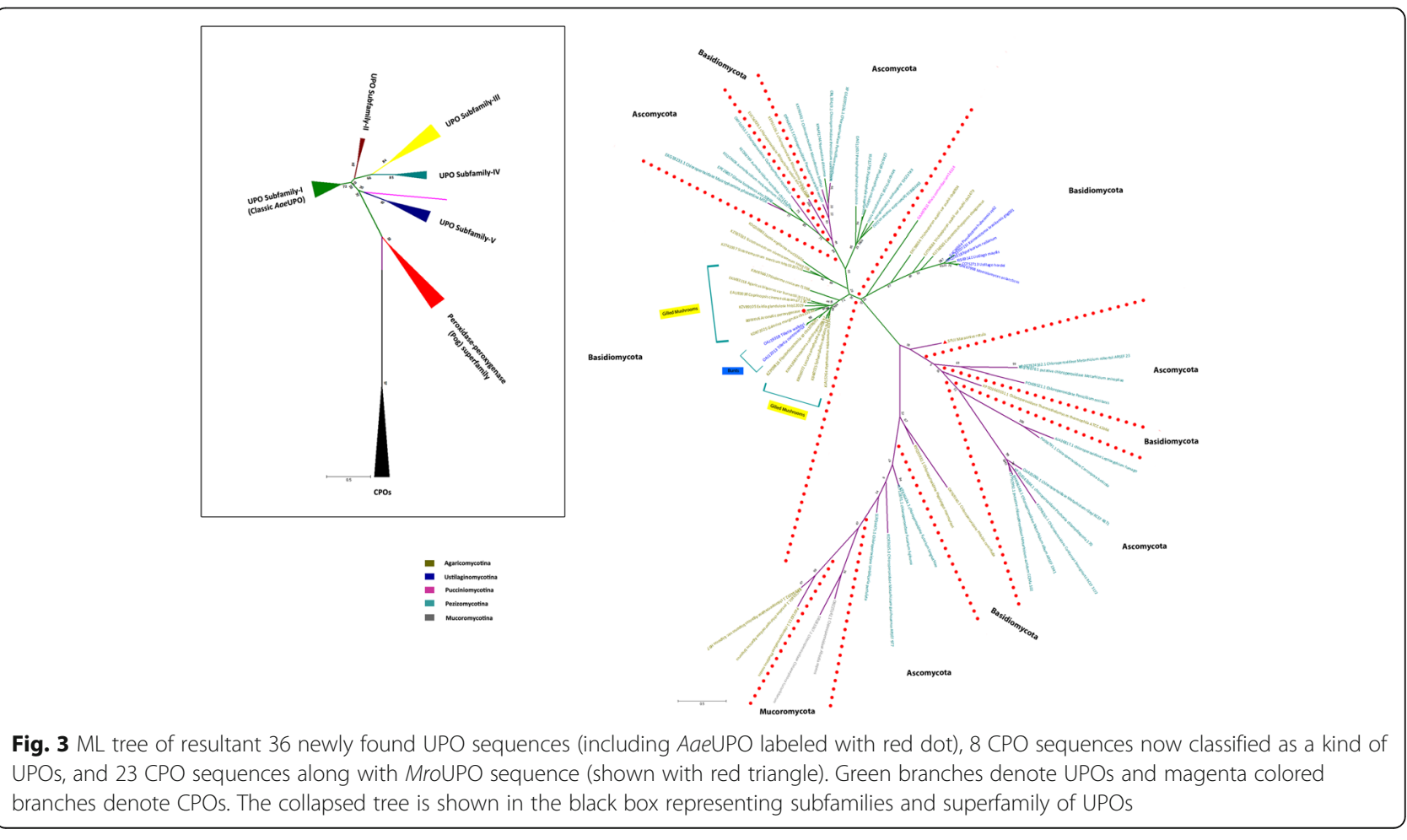


var burnettii jb137s8, which supports the previously proposed hypothesis that Agaricus bisporus sp. may have evolved metabolic strategies and niche adaptations which are lacking in white-rot and brown-rot fungi, and they may also have a distinct distribution of substrate conversion enzymes in adaptation to ecological niche [24]. This could be a possible reason for the presence of both CPO and UPO in Agaricus bisporus sp.

Interestingly, some of the $\mathrm{CPO}$ sequences are placed among four UPO encoding sequences: Glarea lozoyensis atcc20868, Aureobasidium melanogenum cbs110374, Aureobasidium namibiae cbs147.97, and Neonectria ditissima which belong to Pezizomycotina subphylum of Ascomycota suggesting their similar catalytic behavior. The phylogenetic tree shows high scores for these CPO sequences placed among the UPOs. They also have a similar motif pattern as the UPOs with acid-base catalyst required for UPO activity exhibiting EAD/ETD motif in some sequences instead of EGD motif. It is also worth noting that these residues are not defined for $\mathrm{CPO}$ activity as well. Besides, there is no report implicating the CPO activity in these sequences which provide a strong evidence that these CPOs may be another kind of
UPO mistakenly recognized as CPOs. However, the MroUPO is placed along with the $L f u C P O$ and some other $\mathrm{CPO}$ sequences in the phylogenetic tree.

\section{Conserved motifs in UPOs}

The multiple sequence alignment (MSA) (see Additional file 2: Figure S1) and structural analysis of all predicted structures revealed that the resultant UPO sequences consist of the motifs (PCP---EGD---R----E) (Fig. 4) required for the enzyme activity and the binding pocket is surrounded by the aromatic amino acid residues (see Additional file 3: Table S2) as exhibited by AaeUPO.

Our analysis led to the discovery of two new conserved motifs, which are virtually present in all UPOs, namely, the S [IL] G motif located between the PCP and the EGD motifs and SXXRXD motif present after the EGD motif, except in MroUPO. All UPOs consist Ile in S [IL] G motif except three species: Jaapia argillacea mucl33604, Mixia osmundae iam14324, and Sphaerulina musiva so2202, which contain Leu in place of Ile. Strangely, three of these species belong to different subphyla, namely Agaricomycotina, Pucciniomycotina, and Pezizomycotina belonging to both Basidiomycota phylum and Ascomycota phylum as well. Another
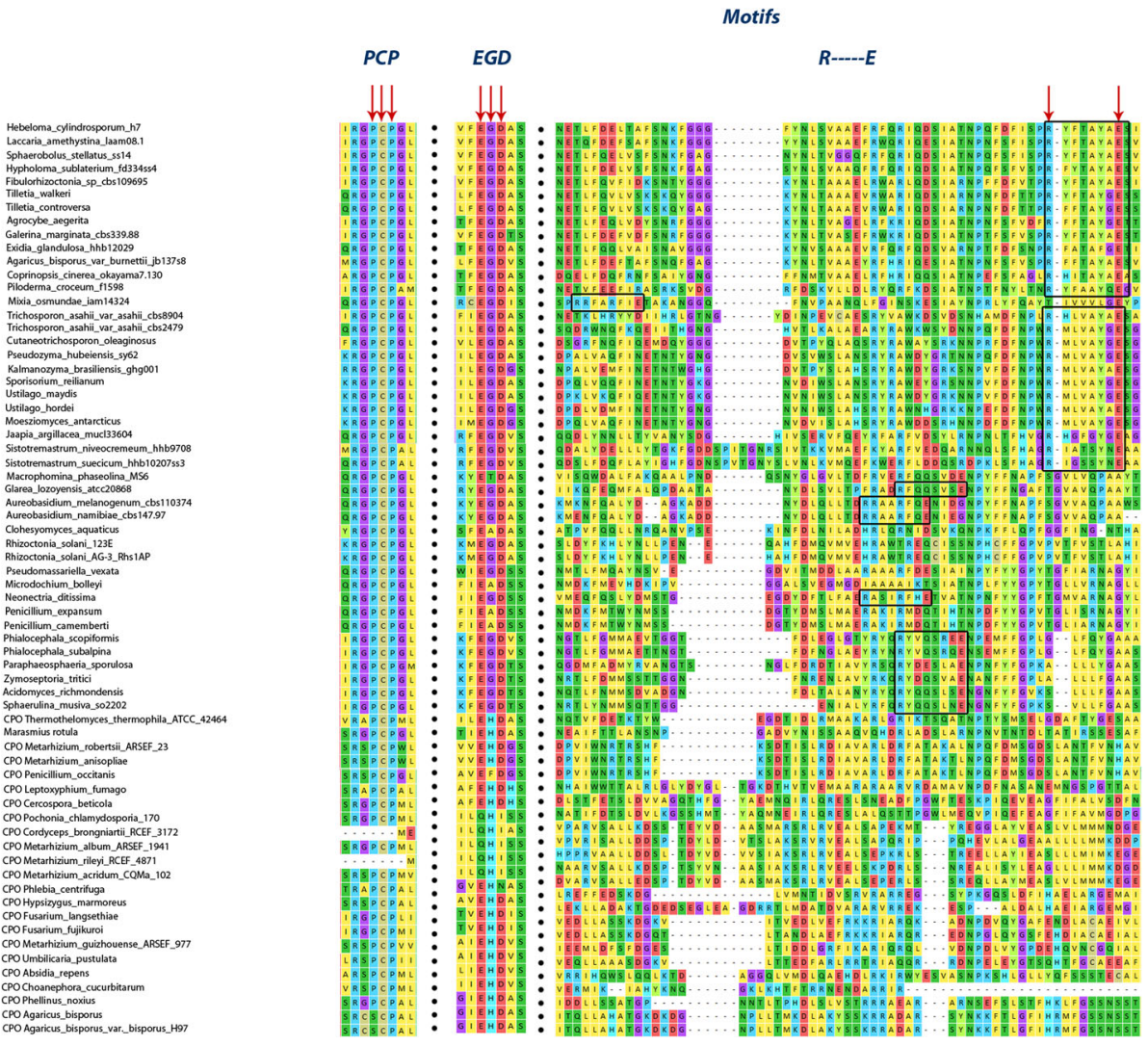

Fig. 4 MSA showing the basic previously known motifs present in all resultant UPOs: PCP---EGD---R------E 
important pattern which we have observed is the presence of six amino acids in between the acid-base catalyst pair in all UPOs (Fig. 4) except some CPO sequences (Clohesyomyces aquaticus, Rhizoctonia solani 123E, Rhizoctonia solani AG-3 Rhs1AP, Microdochium bolleyi, Penicillium camemberti, and Penicillium expansum) placed among the UPOs, which seem to have seven amino acids (see Additional file 2: Figure S1). Besides, some of the new motifs (see Additional file 4: Figure S2) have also been found in different species of UPOs and formed a basis for their classification into five different subfamilies and a superfamily which encompasses both peroxygenase (MroUPO) and peroxidase ( $L f u C P O$ and other $\mathrm{CPO}$ sequences) named as Peroxidase-peroxygenase (Pog) superfamily.

\section{Classification of UPOs and CPOs}

According to the phylogeny analysis, the CPOs which are considered as outgroup are classified into two families: classic CPOs and Pog superfamily, additionally, on the basis of newly found motifs, we classify UPOs into five different subfamilies (Fig. 5). Apart from the earlier recognized motifs, each UPO subfamily consists of its own signature motifs except the Pog superfamily which consists of the two experimentally validated species, namely, $L f u C P O$ and MroUPO both of which have shown peroxygenating (comparatively lesser than that of Aae UPO) and peroxidase activities. $L f u C P O$ has been reported to show epoxidation and carbon-carbon bond cleavage [25] but with lesser potential than that of the AaeUPO [10]. The rest of the CPO sequences present in this family have not been explored yet in terms of their activities, therefore, we predict that these sequences in this superfamily may also be capable of showing peroxygenase and peroxidase activities as the $L f u C P O$ and MroUPO. However, a detailed study is required to assess their properties and catalytic activities. The classic CPOs appear far from the UPOs showing the evolutionary

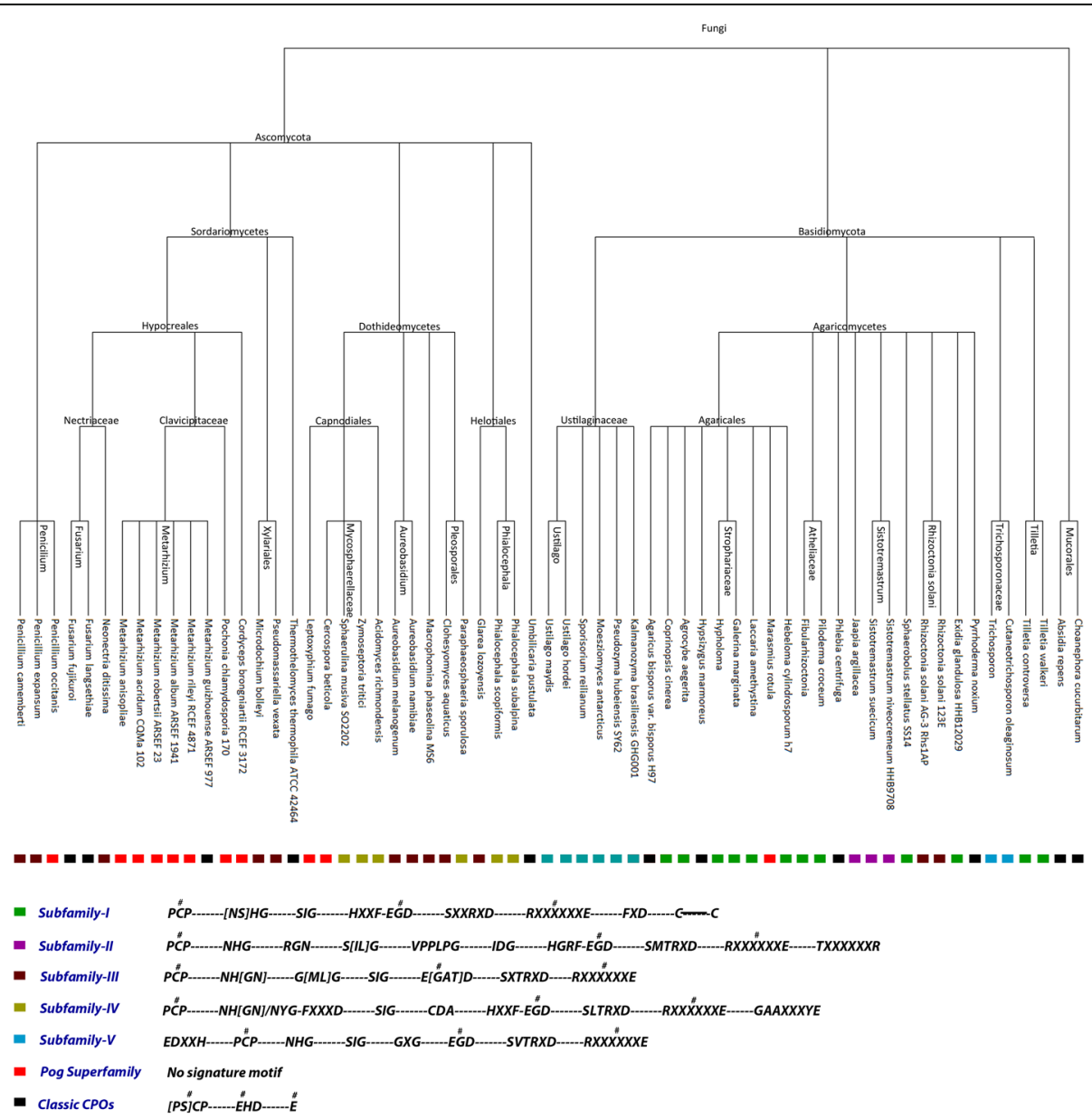

Fig. 5 Species tree consisting of all the fungal species including all UPOs and CPOs belonging to different subfamilies and superfamily along with the motif patterns exhibited by each family. \# signifies the motifs reported earlier in UPOs and CPOs 
distance among them. This family consists of the CPO motifs as reported previously [7].

\section{Selective pressure analyses}

All branches were tested for the selection pressure in UPOs as well as in CPOs. According to the BUSTED method [26], gene-wide episodic diversifying selection in the branches at a $p$-value threshold of $\leq 0.05$ was experienced (see Additional files 5 and 6). The site-specific method identified many functionally relevant sites which have experienced positive diversifying and negative purifying selection in UPOs and CPOs.

\section{Diversifying and purifying selection in UPOs}

The strong purifying and diversifying selection has been detected on UPOs using different models (Additional file 7: Figure S3). Episodic diversifying/positive selection in UPOs at 248 different sites by MEME [27] at a p-value threshold of 0.05 and pervasive diversifying and purifying/negative selection has been identified by FUBAR [28] at 281 and 2 sites respectively at a posterior probability of 0.9 and above (see Additional file 5) (Fig. 6). According to site-specific method (MEME) results, UPOs exhibited synonymous substitutions higher than the nonsynonymous substitutions (see Additional file 8: Figure S4) and 30 functionally relevant sites with episodic and/ pervasive purifying and diversifying selection pressure were recognized from literature discussed as follows: Sites-90, 91, 93, and 100 lie in very close proximity of the PCP motif. Sites-143-145, 147, 149, and 151 showed positive selection which lies in close proximity to residues involved in interacting with the acetate substrate by making Van der Walls interactions between the methyl group and carboxyl $\mathrm{C}$-atom of acetate and pyrrole ring of the heme system in AaeUPO [14]. Sites-184 and 185 in the alignment represent Pro108 and Pro109 undergone positive selection in UPOs which makes a cis-peptide bond with each other in AaeUPO. Also, the sites-280 to 283 and 285 showed diversifying selection. These sites represent the amino acids present between the acid-base catalyst pair in UPOs. Site-200 represents a well-known Gly residue in the EGD motif in UPOs, which has experienced a significant episodic diversifying selection. Sites-284 and 287 showed diversifying selection and site-289 showed purifying selection, all of them are involved in interacting with the aromatic rings in polycyclic aromatic hydrocarbons [14]. Sites396 and 445 represent two Cys residues involved in making a disulfide bridge to stabilize the $\mathrm{C}$-terminal region in AaeUPO [14].

Some of the newly found motifs in the UPOs have also appeared to experience episodic and pervasive selection at different sites. Sites- 74-77 representing the first four residues in EDXXH motif of Subfamily- V UPOs have experienced episodic and pervasive diversifying selection. Site-107 which represents the Gly residue of [SN] HG in Subfamily-I of UPOs, NHG in Subfamily-II, III, and V, and NH [GN]/NYG in Subfamily-IV UPOs. Site- 118, 119, and 120 which represent the first three residues in the FXXXDG motif present in the Subfamily-IV of UPOs showed episodic and pervasive diversifying selection. Sites138 and 140 represents Gly residues in the G [ML] G motif in Subfamily-III of UPOs having experienced

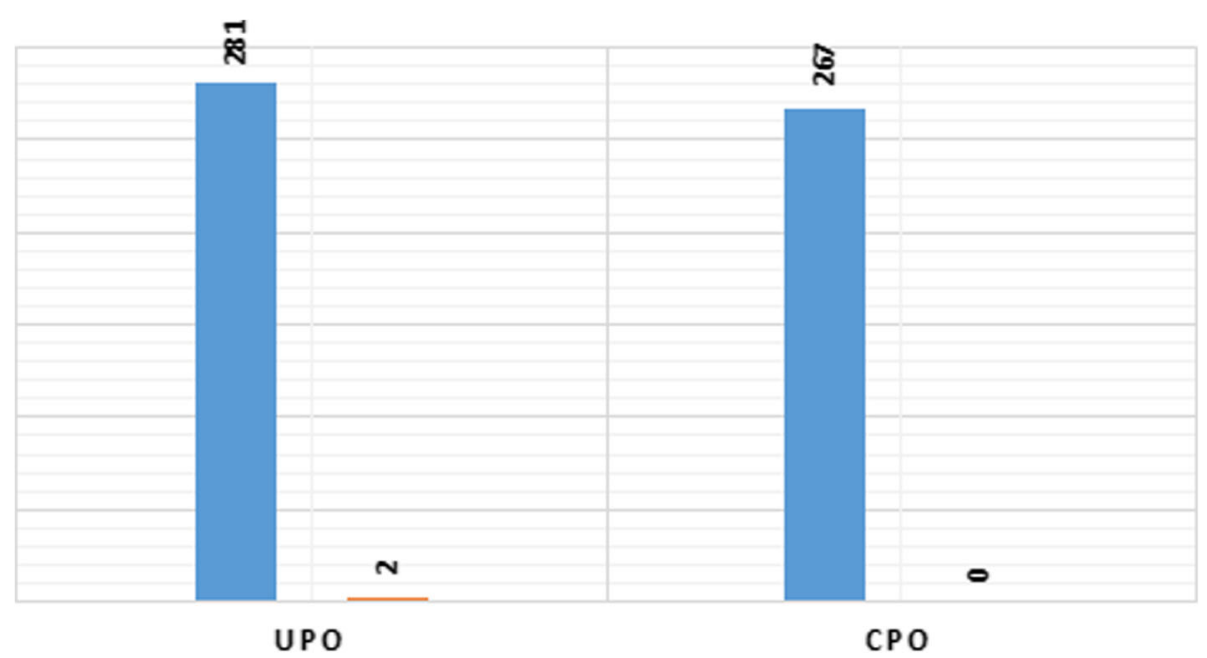

\#positive sites \# \#negative sites

Fig. 6 Bar chart showing a number of sites under positive and negative neutral selection in UPOs and CPOs 
episodic and pervasive diversifying selection. Sites169 and 171 represent Cys and Ala residue in CDA motif present in Subfamily-IV of UPOs and showed episodic and pervasive diversifying selection. Sites-175 to 179 representing the last five residues in VPPLPG motif of Subfamily-II of UPOs showed pervasive and episodic diversifying selection. Similarly, sites- 182 to 184 which represent IDG motif of the same subfamily, have shown diversifying selection. Sites- 188 and 89 appear to have experienced episodic diversifying selection which represent the first and second Gly residue of GXG motif in Subfamily-V UPOs. Site- 197 represents the third residue in HXXF motif of Subfamily-I, II, and IV of UPOs. Sites- 204 and 207 representing amino acid residues present in the SXXRXD motif present in all UPOs showed diversifying selection. Sites- 285, 287-292 showed episodic and pervasive diversifying selection, where site- 289 showed pervasive purifying selection, represent the amino acid residues of GAAXXXYE motif present in Subfamily-IV of UPOs. Site- 295 represents the middle residue of FXD motif in Subfamily-I of UPOs. Sites- 335 to 338 and 341 represent amino acid residues in TXXXXXXR motif of Subfamily-II of UPOs showed episodic and pervasive diversifying selection.

The diversifying selection is seen on a large number of sites in the UPO alignment which indicates the spreading of more beneficial alleles throughout the sequences and also purifying selection which removes deleterious mutations [29].

The branch-site method (aBSREL) [30] found an evidence of episodic diversifying selection on 35 out of 36 tested branches in the phylogeny of UPOs at a $p$-value threshold of $\leq 0.05$. The model fitted to the data with log likelihood are shown in Additional file 5 and the branches showing positive selection are shown in Additional file 9: Figure S5. The positive diversifying selection was detected on all tested branches except the three: Aureobasidium melanogenum cbs110374 which belongs to Pezizomycotina subphylum of Ascomycota, CPO Rhizoctonia solani 123E, and CPO Rhizoctonia solani AG-3 Rhs1AP.

\section{Diversifying and purifying selection in CPOs including MroUPO}

In the case of CPOs, episodic diversifying selection has been identified at 166 different sites by MEME at a p-value threshold of 0.05 and pervasive diversifying and purifying selection has been identified by FUBAR at 267 and 2 sites respectively at a posterior probability of 0.9 and above (see Additional file 6 \& Additional file 10: Figure S6) (Fig. 6). Similar to UPOs, CPOs also showed large number of synonymous substitutions greater than the nonsynonymous substitutions (see Additional file 11: Figure S7). We found 29 functionally relevant sites under positive selection, discussed as follows: Sites-95and 99 to 103 showed episodic and pervasive diversifying selection which are present right before and after the $\mathrm{PCP}$ motif respectively and are involved in providing rigid scaffolding for iron-sulfur interactions in $L f u C P O$ [31], and encompasses conserved Cys residue present in all HTPs and stabilizes the C-terminal region [7]. Sites-80 and 369 showing diversifying selection function as $\mathrm{N}$-glycosylation sites in $\mathrm{LfuCPO}$. Site-96 represents the Pro in the PCP motif in CPOs which appeared to have experienced the episodic diversifying selection. Sites- 185 and 193 represent two Cys residues which form a disulfide bond in LfuCPO. A Ser residue at site-216 showing episodic diversification forms a loop with the Glu residue of EHD motif in CPOs providing a primary set of interaction. Sites-206-208, 213, and 216 along with site-433 showed diversifying selection which function in forming a small channel connecting the heme distal site in CPOs [31]. Sites-209, 311, and 383 are identified with diversifying selection and these sites make interactions with organic substrates such as dimethylalanine which undergoes CPO-catalyzed oxidative demethylation [32]. Sites-392, 393, 395, 396, 403 to 405, 439, and 447 showed diversifying selection and are involved in binding with carbohydrates [31].

The branch-site method, aBSREL found evidence of episodic diversifying selection on 26 branches out of 31 branches tested as foreground in the phylogeny of CPOs at a $p$-value threshold of $\leq 0.05$ (see Additional file 6). The model fitted to the CPO data with log likelihood are shown in Additional file 5. The branches showing positive selection are shown in Additional file 12: Figure S8. The branches with selection evidence include Marasmius rotula, Metarhizium guizhouense ARSEF 977, Metarhizium album ARSEF 1941, Penicillium occitanis, Pochonia chlamydosporia 170, Phellinus noxius, Penicillium expansum, Leptoxyphium fumago, Umbilicaria pustulata, Thermothelomyces thermophila ATCC 42464, Pseudomassariella vexata, Clohesyomyces aquaticus, Macrophomina phaseolina MS6, Phlebia centrifuga, Choanephora cucurbitarum, Metarhizium rileyi RCEF 4871, Microdochium bolleyi, Metarhizium anisopliae, Cordyceps brongniartii RCEF3172, Fusarium langsethiae, Cercospora beticola, Absidia repens, Metarhizium acridum CQMa 102, Hypsizygus marmoreus, Penicillium camemberti, and, Fusarium fujikuroi. The negatively selected branches include Metarhizium robertsii ARSEF 23, Agaricus bisporus, and Agaricus bisporus var. bisporus $H 97$.

\section{Functional divergence analyses}

Type-I functional divergence (also known as site-specific rate shift) was observed in all the pairs 
but no significant radical (Type-II) divergence was observed (Table 1). The sites in all clusters show the typical pattern of Type-I functional divergence which signifies the distribution of diverse amino acid residues in one cluster (tested cluster) and conserved amino acid residues in another cluster (background cluster) (see Additional file 13: Figure S9). 13 functionally relevant sites have been identified with a significant posterior probability in UPOs as well as in CPOs showing the Type-I functional divergence (Fig. 7). The Type-I analysis on the clusters revealed that the CPOs are more conserved than the UPOs whereas the Basidiomycota UPOs and Ascomycota UPOs showed functional divergence with respect to CPOs along with MroUPO (Fig. 8).

\section{Type-I functional divergence (site-specific rate shift) in UPOs}

UPOs were observed to exhibit shifted functional constraints at the following sites: Sites-109 to 112 lies close to the PCP motif, site-150 and 155 are present close to the residues involved in making van der Waal's interaction with acetate substrate in UPOs. Sites- 148-150 which signify the newly found motif G[ML]G in the Subfamily-IV UPOs, showed significant functional divergence with the posterior probability of $0.32,0.47$, and 0.88 respectively. Sites166 and 167 show functional divergence with the posterior probability above 0.9 in the Basidiomycota UPOs, where these sites represent the Gly and Asn in the RGN motif in Subfamily-IV of UPOs. Sites216-218 signify GXG motif in Subfamily-V of UPOs and showed significant values for site-specific rate shift in Basidiomycota and Ascomycota UPOs. The SXXRXD motif found in all UPOs also showed significant functional divergence at all of its residues except the first Ser. The sites-226, 234, 235, and 236 lies in close proximity to the EGD motif. The Gly residue in EGD motif in Ascomycota UPOs showed a significant functional divergence with a posterior probability of 0.66 which is involved in site-specific rate shift UPOs from the CPOs.

\section{Type-I functional divergence (site-specific rate shift) in CPOs}

Ascomycota UPOs vs CPOs including MroUPO pair shows a Type-I functional divergence at several functionally relevant sites such as sites-217-221 and 223-226 which lie close to the EHD motif in CPOs (including MroUPO) with a posterior probability ranging between $0.5-09$. Most importantly, this pair showed a significant functional divergence at the site-228, which represents the His and Gly residues in CPOs and Ascomycota UPOs respectively with a posterior probability of 0.6 . Another functionally relevant site includes site-227 which makes a hydrogen bond with $G l n$ located at a distance and maintains its orientation and provides charge-charge interactions.

\section{Type-I analysis}

The Type-I Analysis shows site-specific profile predicting critical amino acid residues responsible for site-specific rate shift in the enzymes (see Additional file 14). According to the results, the CPOs cluster has not shown any divergence but the other two clusters, i.e., Basidiomycota UPOs and Ascomycota UPOs have shown some specific sites with significant posterior probabilities (Fig. 8).

\section{Ascomycota UPOs}

The Ascomycota UPOs showed 8 specific residues which have experienced functional divergence with respect to the CPOs and may have been functionally relevant in their catalytic roles. Site-134 shows a consistent pattern of Ile/ $\mathrm{Val} / \mathrm{Leu}$ in the CPOs but showed substitutions in the Ascomycota UPOs replaced by Val/Thr/Ala/Ser/Gly. Similarly, another site-166 shows the presence of only two residues, namely, Gly/Ala in Basidiomycota UPOs and no particular residues in Ascomycota UPOs and CPOs. Site-217 shows the conserved Leu in CPOs but no particular distribution of residues in Ascomycota UPOs. Site-218 shows the presence of Gly in Ascomycota UPOs as well as in Basidiomycota UPOs but no specific residues in CPOs. Similarly, site-219 shows Leu/ Ile residues in Ascomycota UPOs and Basidiomycota UPOs but any such specific residues are not present in CPOs at this site. Site-232 does not show a particular residue distribution but it lies in close proximity to EGD and/or EHD motif in UPOs and CPOs respectively. Site-261 represents the conserved Gly residue in Ascomycota as well as in Basidiomycota UPOs but no specific residues in CPOs were found distributed except in the MroUPO which also consists of Gly at the same site. Site-262 shows the distribution of Asn/Asp in Basidiomycota UPOs, Asn/Asp/Gln in Ascomycota UPOs, and no specific pattern in CPOs.

Table 1 Showing the $\theta_{\mid}$and $\theta_{\|}$values of all the pairs studied

\begin{tabular}{|c|c|c|c|c|c|}
\hline Species & $\theta_{I} \pm \theta_{\mathrm{SE}}$ & $\Theta_{\|} \pm \theta_{\mathrm{SE}}$ & \#Sites with no change & \#Sites with radical change & \#sites with conservative change \\
\hline Basidiomycota- vs Ascomycota- UPOs & $0.048048 \pm 0.073911$ & $-1.788713 \pm 0.819243$ & 39.411765 & 18.705882 & 14.882353 \\
\hline Basidiomycota UPOs vs CPOs & $0.342599 \pm 0.062568$ & $-0.902184 \pm 1.326494$ & 27.352941 & 27.705882 & 17.941176 \\
\hline Ascomycota UPOs vs CPOs & $0.387130 \pm 0.084127$ & $-1.640309 \pm 1.841228$ & 26.764706 & 27.941176 & 18.294118 \\
\hline
\end{tabular}


Type-I

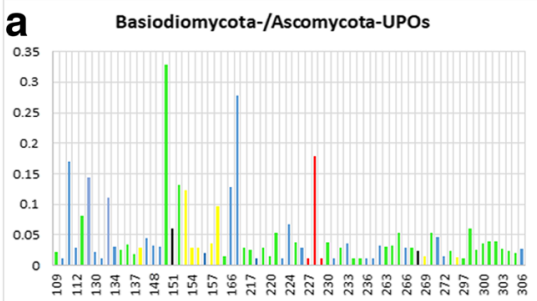

$\uparrow$

b Basiodiomycota-UPOs/CPOs + MroUPO

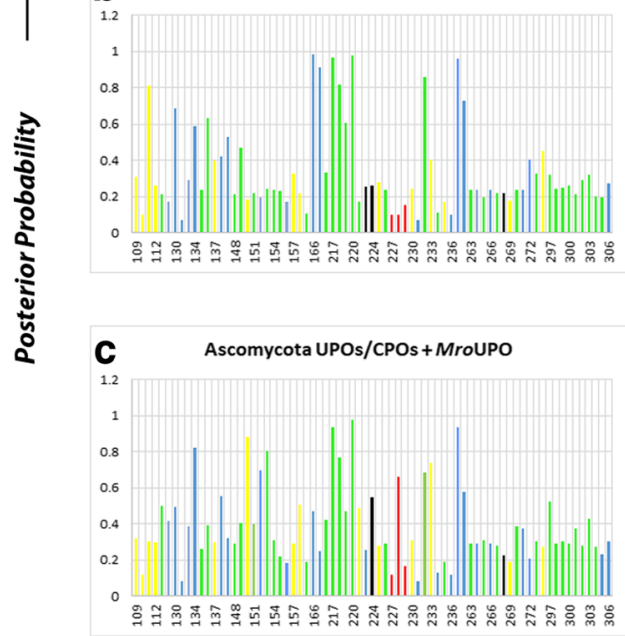

Type-II
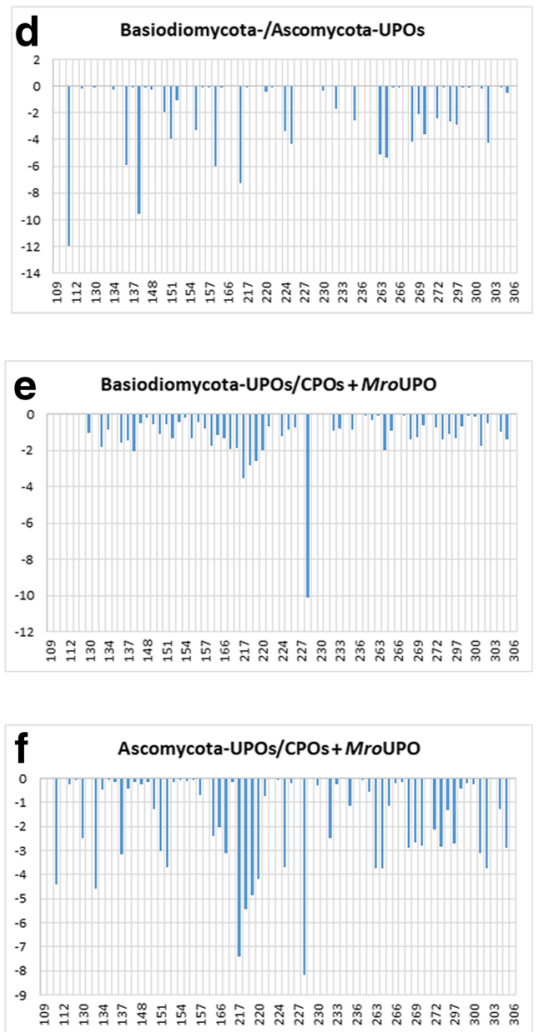

Sites of residues in alignment

Fig. 7 Column graphs showing Type-I (a-c) and Type-II (d-f) functional divergence analysis on CPOs and UPOs. Red bars represent the sites having EGD motif in UPOs and EHD motif in CPOs, the black bar represents the significant and functionally relevant sites, green bars indicate the positively selected sites showing functional divergence, yellow bars indicate the sites showing positive selection along with functional divergence, and magenta colored bars indicate sites showing negative selection

\section{Basidiomycota UPOs}

Basidiomycota UPOs have shown 2 specific residues which are involved in functional divergence. One of these sites, site-228 represents the Gly residue in the UPOs and His residue in CPOs and MroUPO. Another site-150 shows the presence of Gly/Ala in Ascomycota UPOs but not any specific distribution of residues in CPOs were found at the same site.

\section{Discussion}

\section{Distribution and functional evolution of fungal UPOs}

Fungal species exhibiting UPO identified in the subphyla of Basidiomycota and a single subphylum of Ascomycota appear very diverse in their characteristics, habitats, and behaviors. Among the obtained UPO sequences, three species belonging to Pezizomycotina are plant pathogenic: Neonectria ditissima causes cankers in Apples [33], Zymoseptoria tritici causes leaf blotch in wheat [34], and Sphaerulina musiva causes leaf spot and canker disease in poplar trees [35]. Besides, UPO sequences were also found in some extreme environment surviving species such as Aureobasidium melanogenum cbs110374, A. namibiae cbs147.97, which can tolerate up to $10 \% \mathrm{NaCl}$ and grows between 10 and 35 degree Celsius [36], Acidomyces richmondensis which is adapted to extremely acidic $(\mathrm{pH}<1)$ and thermophilic (40-50\%) environment of acid mine drainage [37], and Phialocephala subalpina, which is a dark septate endophyte that increases plant tolerance against salt or drought [38]. The species belonging to Ustilaginomycotina are mostly plant parasitic in nature. Tilletia walkeri also known as Ryegrass bunt and $T$. controversa both are plant pathogens. Other pathogenic species belonging to Ustilaginomycotina include Ustilago maydis, $U$. hordei which cause corn smut on maize and barley respectively [39], and Sporisorium reilianum which infects maize and sorghum leading to head smut in both [40]. Another pathogenic species found to have UPO is Mixia osmundae 


\section{Type-I Analysis}

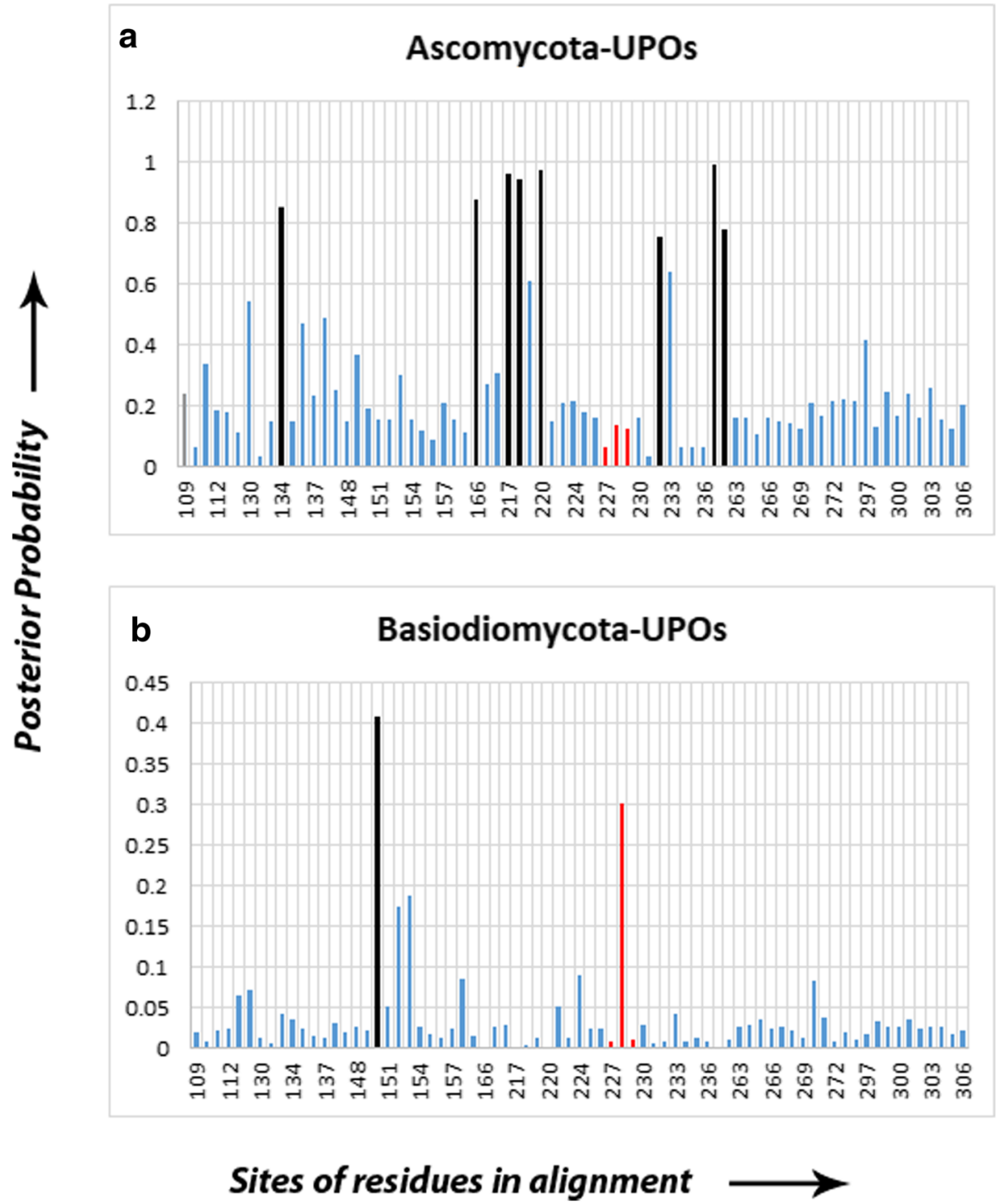

Fig. 8 Column graphs showing the critical amino acids involved in functional divergence in (a) Ascomycota UPOs and (b) Basidiomycota UPOs. Red colored bars indicate the EGD motif in UPOs and black bars represent the newly found critical sites which are involved in functional divergence

iam14324 which is an intracellular parasite of ferns, belongs to Pucciniomycotina. Interestingly, none of the fungal species consisting of UPO obtained in this study is edible. Aureobasidium melanogenum cbs110374 also known as Aureobasidium pullulans var melanogenum cbs110374 is also responsible for causing infections in humans [36]. Besides, the A. pullulans infection potential is partially linked to the production of some extracellular enzymes [41]. However, Glarea lozoyensis atcc20868 has medical relevance due to its ability to produce natural antifungals named pneumocandins, which act by inhibiting fungal $\beta-(1,3)$-glucan synthesis [42] and has provided antifungal therapy to treat life-threatening fungal infections [43]. Another non-pathogenic fungal species having UPO is Pseudozyma hubeiensis sy62 which produces a large number of extracellular glycolipids, saccharides, and mannosylerythritol lipids from vegetable oils. The different characteristics and behavior of the fungal species having UPOs show their wide and diverse distribution in the fungal kingdom.

\section{Hypothesized functional roles of newly found conserved motifs in UPOs}

As implicated by earlier publications, the UPOs have three conserved motifs: PCP, EGD, and R-E. Here, we have found some new conserved motifs among different 
subfamilies of UPOs and mapped them on to the experimentally resolved structure of AaeUPO including the other modeled structures of newly found UPOs (Additional file 15: Figure S10) revealing close proximity to the binding site in the enzymes. Therefore, we postulate that they may play an important role in substrate binding and specificity. These motifs are discussed as follow: NHG/NHN/SHG motif may be responsible for actively participating as the active and binding residues as Asn, His, and Gly are supposed to be involved as the binding residues in proteins, and $\mathrm{Ser}$ is capable of forming H-bonds with polar substrates. The S [IL] G motif lying in between the PCP and the EGD motifs. This SIG motif lies in close proximity to the binding pocket of the enzyme. We predict that the role of this motif might be related to its specificity for the substrates as Ser is slightly polar in nature capable of forming hydrogen bonds with various polar substrates, and Gly contains a hydrogen as its side chain which could provide conformational flexibility, and Ile is an aliphatic hydrophobic amino acid which could be involved in the binding and recognition of hydrophobic ligands such as lipids [44]. However, the above mentioned three species consists of Leu instead of Ile, which is also hydrophobic in nature and may be involved in as the same function as the Ile. The SXXRXD motif in all UPOs may be responsible in providing stability to the structure and forming $\mathrm{H}$-bonds with a variety of polar substrates as Ser, Thr, and Asp are involved in forming $\mathrm{H}$-bonds with the polar substrates and to provide protein stability. Another conserved sequence motif which was recognized through sequence analysis of all UPOs was the presence of six amino acids in between the acid-base catalyst pair. The occurrence of the PCP and EGD motifs is same in all fungal species consisting of UPO but the species from different subphyla follow a different pattern of amino acid residues arrangement present in between the acid-base catalyst pair except for two species Tilletia walkeri and Tilletia controversa from Ustilaginomycotina, which follows more similar pattern as the Agaricomycotina UPO consisting species than those belonging to Ustilaginomycotina of Basidiomycota (see Additional file 2: Figure S1). However, there is no consistent presence of a specific pattern of amino acid residues in between the acid-base pair. The hypothesized functions of the UPOs belonging to different subfamilies are summarized in Table 2 based on the roles of amino acid residues present in the motifs.

\section{Phylogenetic inference of UPOs}

The phylogeny of UPOs suggests that most of the highly similar UPOs with respect to AaeUPO belong to Agaricomycotina followed by Ustilaginomycotina, and Pucciniomycotina subphyla of Basidiomycota and Pezizomycotina subphylum of Ascomycota. However, four of the resultant UPOs (Glarea lozoyensis atcc20868, Aureobasidium melanogenum cbs110374, Aureobasidium namibiae cbs147.97, and Neonectria ditissima) that belong to Ascomycota are

Table 2 Hypothesized functions of the classified subfamilies of UPOs based on the roles of amino acid residues present in the motifs

\begin{tabular}{|c|c|c|c|}
\hline Subfamily & Motif & ${ }^{a}$ Roles of amino acids present in the motif & Hypothesized functions of the subfamily \\
\hline \multirow[t]{2}{*}{1} & FXD & $\begin{array}{l}\text { Phe is basically involved in stacking interactions with other aromatic side- } \\
\text { chains and the Asp is frequently involved in salt-bridges interacting with } \\
\text { positively charged amino acids to create stabilizing } \mathrm{H} \text {-bonds which can be } \\
\text { important for protein's stability. }\end{array}$ & $\begin{array}{l}\text { may actively involve in interacting with aromatic residues } \\
\text { and in forming stable } \mathrm{H} \text {-bonds imparting to the structural } \\
\text { stability. }\end{array}$ \\
\hline & Cys-Cys & disulfide bond is mostly involved in providing stability to protein structure. & \\
\hline \multirow[t]{3}{*}{$\|$} & RGN & $\begin{array}{l}\text { Arg is frequently involved in making salt-bridges with the negatively charged } \\
\text { amino acids creating stable H-bonds which may be crucial for the structure } \\
\text { stability; the Gly provides the conformational stability, and the Asn is in- } \\
\text { volved as protein's active and binding sites. }\end{array}$ & $\begin{array}{l}\text { may potentially interact with the hydrophobic ligands such } \\
\text { as lipids and may show specificity for some polar substrates. }\end{array}$ \\
\hline & IDG & $\begin{array}{l}\text { Ile in the IDG motif is involved in recognizing hydrophobic ligands; Asp } \\
\text { forms stable H-bonds with positively charged amino acids required for pro- } \\
\text { tein's stability, and the Gly again may provide conformational stability. }\end{array}$ & \\
\hline & TXXXXXXR & $\begin{array}{l}\text { Thr is often found in protein centers and capable of forming } \mathrm{H} \text {-bonds with } \\
\text { the polar substrates. }\end{array}$ & \\
\hline III & $\mathrm{G}[\mathrm{ML}] \mathrm{G}$ & $\begin{array}{l}\text { the Gly provides the conformational stability, Met and Leu plays a role in } \\
\text { binding and recognition of hydrophobic ligands. }\end{array}$ & $\begin{array}{l}\text { may play important role in substrate specificity/recognition } \\
\text { and capable of forming strong } \mathrm{H} \text {-bonds with the polar } \\
\text { substrates. }\end{array}$ \\
\hline IV & $\begin{array}{l}\text { CDA, FXXXDG, } \\
\text { GAAXXXYE, and } \\
\text { HXXF }\end{array}$ & $\begin{array}{l}\text { Ala is involved in substrate recognition and specificity; Tyr makes stacking } \\
\text { interactions with the aromatic side chains; His is involved in protein metal } \\
\text { binding sites; and Phe also makes stacking interactions with aromatic side } \\
\text { chains. }\end{array}$ & $\begin{array}{l}\text { may show large interactions with the aromatic substrates } \\
\text { and these motifs are perhaps involved in substrate } \\
\text { recognition and binding. }\end{array}$ \\
\hline \multirow[t]{2}{*}{ V } & EDXXH & $\begin{array}{l}\text { His is most commonly involved in active and binding sites especially in } \\
\text { metal binding sites and the Asp and Glu residues create the stable } \mathrm{H} \text {-bonds. }\end{array}$ & $\begin{array}{l}\text { may play an important role in reacting with positively } \\
\text { charged amino acids. }\end{array}$ \\
\hline & GXG & Gly provides the conformational stability & \\
\hline
\end{tabular}


placed in a cluster along with CPOs in the phylogenetic tree which indicates that they may show less AaeUPO-like activity than that of MroUPO and LfuCPO. Besides, the MroUPO is placed in between the Ascomycota UPOs and the Ascomycota CPOs all of which belonging to Pezizomycotina subphylum. This clearly indicates the intermediate state of the MroUPO existing between the CPOs and UPOs. However, these CPOs belong to the Pog superfamily which surpasses both peroxygenase and peroxidase suggesting that the other $\mathrm{CPO}$ sequences may also exhibit peroxygenase properties. Additionally, some of the $\mathrm{CPO}$ sequences are placed along with UPOs, which on further analysis, appeared more like a kind of UPOs.

Most of the resultant highly similar UPOs are "gilled mushrooms" which are most commonly found in nature. It is worth noting that only a single UPO species i.e., Mixia osmundae iam14324 is present in Pucciniomycotina and most of the fungal species exhibiting UPOs are plant pathogenic and/or harmful in nature. The most highly similar fungal species to AaeUPO, i.e., Galerina marginata cbs339.88 is extremely poisonous species from the Hymenogastraceae family of the order Agaricales. Although Aae UPO is not plant parasitic fungus, the majority of the other obtained UPOs are pathogenic in nature which may indicate an early evolutionary origin of UPOs. It also suggests that UPO is widespread in Basidiomycota of fungal kingdom exhibiting different kinds of species showing variant behavior and adaptations. Besides, the Agaricus genus found consists of CPO and UPO in different strains which may be linked to their adaptable behavior.

\section{Diversifying and purifying selection among UPOs and CPOs}

The UPOs and CPOs are very distant in terms of their divergence which may result in the saturation of $d S$. Therefore, to avoid this, we analyzed a large set of sequences of UPOs (44 sequences) and CPOs (23 sequences) that helped in providing more reliable information. Secondly, terminal branches were selected for $\mathrm{dS}$ and $\mathrm{dN}$ estimation avoiding the interspecific branch lengths. Besides, the higher $\mathrm{dN} / \mathrm{dS}$ ratio detected at a relatively lower level of divergence $(\mathrm{dS}$ and $\mathrm{dN}<0.1)$ provides a strong evidence that the higher inferred intensity of selection in UPOs and CPOs was not caused by the saturation of $\mathrm{dS}$.

A strong diversifying and purifying selection was observed at various functionally relevant sites in UPOs and CPOs. These sites include Gly in the NHG/SHG/NHN motif in all UPOs, EDXX in the EDXXH motif found only in Subfamily-V UPOs, Gly in the G[ML]G motif found in Subfamily-III UPOs, IDG motif of Subfamily-II UPOs, FXX and Cys and Ala in the FXXXDG and CDA motif in Subfamily-IV UPOs respectively, the Gly residue in the well-known EGD motif in all UPOs, and residues present between the acid-base catalyst. These motif sites have experienced strong diversifying selection indicating the spread of beneficial alleles and given the supposition of their roles in substrate specificity, recognition, and structure stability, they may have also stabilized the function of catalyzing a specific substrate among the UPOs. On the other hand, in CPOs, several sites which are relevant in stabilizing the structure and substrate binding have experienced diversifying selection. We postulate that these positively selected sites in UPOs and CPOs have significantly contributed to the evolution of their functional diversity.

\section{Functional divergence among UPOs and CPOs}

The proportion of fixed radical change $(F 00, R)$ and conserved change $(\mathrm{F} 00, \mathrm{C})$ was zero in all pairs indicating a radical functional conservation in UPOs and CPOs. The Basidiomycota UPOs cluster showed site-specific rate shift with respect to $\mathrm{CPOs}$ and MroUPO cluster, and same was followed by the Ascomycota UPOs showing even higher values of functional divergence including the EGD and EHD motifs in UPOs and CPOs respectively. The former cluster showed a very small value (0.09) of Type-I functional divergence on His/Gly residues in EHD/EGD motifs as compared to the latter (0.62) indicating Basidiomycota UPOs as more conserved as compared to the Ascomycota UPOs. According to the Type-I Analysis, CPOs cluster has not shown any functional divergence but was shown by Basidiomycota UPOs and Ascomycota UPOs with respect to CPOs including MroUPO, which confirms the divergence of UPOs from CPOs without any rapid evolution. Besides, some sites were predicted with higher values of posterior probabilities other than the EHD motif in CPOs, which indicates that there are relevant sites other than the EHD/EGD motif which might be responsible for different catalytic activities of CPOs and UPOs (Fig. 8).

\section{Intermediate behavior of MroUPO ranging between UPO and $\mathrm{CPO}$}

Among the predicted critical amino acid residues in Ascomycota UPOs and Basidiomycota UPOs (posterior probability ranging between $0.8-0.9$ ), most of the residues in MroUPO followed as the same pattern as the CPOs. For example, site-134 in CPOs showed Ile/Val/ Ala/Gly and MroUPO exhibits an Ile at this site while none of the UPOs from Basidiomycota or Ascomycota exhibits an Ile. At another site-217, both CPOs and MroUPO consist a Leu but no specific pattern in UPOs. Site-218 exhibits a conserved Gly in UPOs but no such 
residue exists in CPOs and MroUPO. Similarly, site-219 consists of Leu/Ile in UPOs but not in CPOs and MroUPO. Only one such site in MroUPO resembled the pattern of UPOs at the site-261 identified at a posterior probability of 0.99 , which also consists of a conserved Gly in UPOs and in MroUPO as well. The function of these sites is unknown but may be responsible for the distinct behavior of AaeUPO, LfuCPO, and MroUPO.

\section{Correlation between positive/diversifying selection and functional divergence}

The positive and negative selection sites were also mapped at the sites showing functional divergence in UPOs and CPOs (Fig. 7). Our results show that there are a large number of sites under selection pressure as well as the functional divergence together. These sites including other predicted critical residues in UPOs were later mapped on to the three-dimensional structure of AaeUPO (Fig. 9). Some of these sites interact with the aromatic rings in polycyclic aromatic hydrocarbons [14] and the function of rest of the other sites is unknown. Interestingly, one of these functionally unknown sites, Thr55 in AaeUPO showed positive selection and functional divergence and was as well predicted to be a critical amino acid residue possibly responsible for driving the functional divergence of UPOs from the CPOs. The sites showing selection pressure and functional divergence are majorly distributed around the binding region of AaeUPO and hence, we postulate that they might be involved in making interactions with the substrates. As positive selection leads to the spreading of advantageous mutations, it has been found associated with the protein functional shifts [45]. As evident from the results, Ascomycota and Basidiomycota UPOs clusters showed diverse amino acids in opposition to CPOs cluster, also showing diversifying selection may have resulted into the functional divergence among the UPOs.

\section{Conclusion}

In this study, we have found 113 putative fungal UPOs from 35 different species (including strains) distributed in Basidiomycota and Ascomycota phyla. The single sequences from each species were thoroughly analyzed and studied for their phylogeny along with $\mathrm{CPO}$ and further, analyzed for selective pressure and functional divergence. Here, we report seven novel findings: (1) 113 new putative fungal UPO encoding sequences, (2) five subfamilies of UPOs and a superfamily on the basis of phylogeny and the motif patterns, (3) 16 new conserved motifs present in all and/or subfamilies of UPOs which are hypothesized to be involved in substrate specificity, recognition, and binding, (4) existence of a specific pattern having six amino acid residues in between the acid-base catalyst pair in UPOs, (5) pervasive diversifying and purifying selection among UPOs and CPOs, (6) site-specific functional shift in CPOs and UPOs suggesting their evolution from the CPOs and MroUPO being an intermediary state between the two, and (7) some predicted critical amino acid residues in UPOs other than the known motifs, which could have been responsible for their functional diversity.

However, the main physiological role of UPOs in fungi remains unknown but according to the AaeUPO catalyzed reactions could be bio-physiologically related to some special habitats with high amounts of aromatic compounds and lignocellulose fragments which can be utilized as a carbon source. So far studied UPOs exhibit a vast variety of properties such as AaeUPO and MroUPO. Similarly, newly obtained fungal UPOs may exhibit far more interesting properties. They have a wide range of possibilities in synthetic chemistry such as efficient enantioselective oxidations of bulk and fine chemicals, development of personalized drugs and reference metabolites, and biomimetics. The future works involve the detailed study of gilled mushrooms consisting of
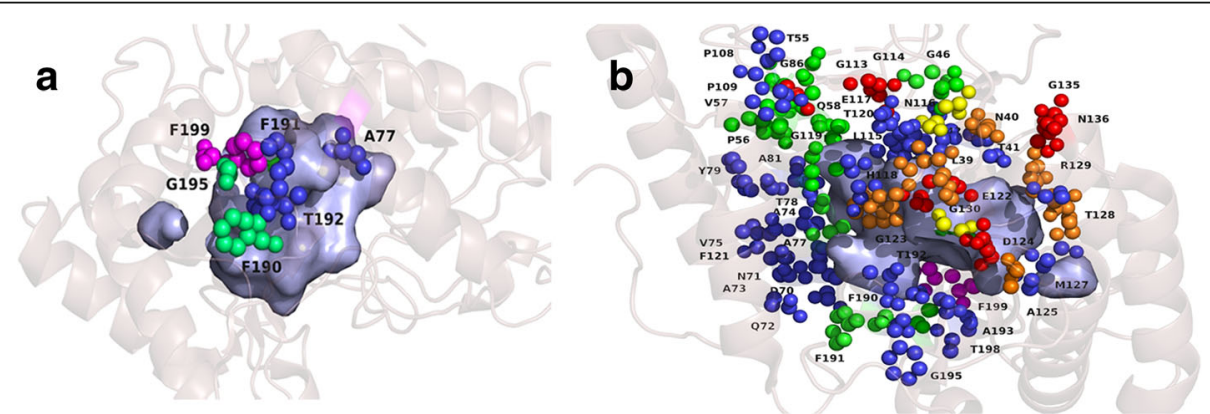

Fig. 9 showing functionally divergent sites with selection pressure in AaeUPO a) showing the residues which interact with aromatic rings in polycyclic aromatic hydrocarbons, and $\mathbf{b}$ ) showing other relevant sites. The surface area represents binding pocket and residue sites are represented as small spheres. Orange spheres show Type-I functional divergence sites, green spheres indicate positive selection, blue spheres indicate the sites with positive selection and Type-I functional divergence, red spheres represent the predicted specific residues responsible for functional divergence in UPOs, yellow spheres indicate residues with positive selection+functional divergence+predicted critical residue and magenta colored spheres indicate negative selection 
UPOs, features of different subfamilies of UPOs, the cause of their similarities and/or differences among them, a comparative study with the other species which are found lacking the UPOs, and a detailed study on the Pog family. This study has provided more UPOs which can be studied further in details to identify the physiological role of UPOs in fungal species.

\section{Methods}

\section{Overview of the analysis pipeline}

Various in-silico filters were applied to mine all the genome sequences for obtaining relevant sequences similar to AaeUPO. The protein crystal structures of the obtained UPO encoding sequences were predicted to analyze their binding pockets. Further, these obtained sequences were subjected to phylogenetic analysis to study their relatedness with CPOs and distribution among the fungal phyla. The resultant phylogeny was analyzed for the signature of Long Branch Attraction (LBA) to avoid any false conclusion regarding the evolutionary relationships. Further, the sequences of obtained fungal UPO species were thoroughly analyzed to study the arrangement of motif patterns and differences among the UPOs belonging to different subphyla. The UPO and CPO sequences were analyzed for selection pressure to identify the positive diversifying or negative purifying selective sites and branches in phylogeny and were subjected to functional divergence study. Further, sites with selection pressure and functional divergence in UPOs were also mapped to the structure of AaeUPO as well as the multiple sequence alignment of all UPOs and CPOs.

\section{Data collection}

Peptide sequences of all fungal genomes constituting 812 different species (or strains) were downloaded from the Ensembl fungal genome database via FTP (ftp:// ftp.ensemblgenomes.org/pub/) [46]. The details of the fungal genomes are provided in Additional file 16: Table S3. The fungal $\mathrm{CPO}$ sequences representing a complete $\mathrm{CPO}$ protein were downloaded from NCBI [47], details are provided in Additional file 17: Table S4.

\section{Homology search and identification of UPO motifs}

We created a pipeline for the identification of UPOs in fungal genomes. AaeUPO was used as a query to perform a similarity search using PHMMER (http://hmmer.org; version 3.1b2) against the generated fungal genomes database with an E-value of 10.0 and an inclusive E-value of 0.01 giving $\sim 1$ false positive in every 100 searches with different query sequences which were further filtered by sequence-based clustering using cd-hit software [48] at $90 \%$ similarity cut-off and a word-length of 5 residues providing representatives of the clustered families. The obtained sequences were further subjected to graph-based clustering using MCL software [49] at an inflation value of 1.4 to eliminate the dissimilar sequences corresponding to the seed sequence. The resultant sequences were then searched for motifs providing the final resultant UPO-encoding sequences. The most highly similar sequences among these obtained putative sequences were selected using ClustalX2 [50] by calculating the similarity of putative sequences belonging to a specific species with respect to the AaeUPO protein sequence.

\section{Structure prediction}

The protein structures of the finally obtained 35 highly similar sequences were predicted using SwissModel [51] and their binding cavities were analyzed for the presence of surrounding aromatic residues as found in AaeUPO using Pymol [52]. The predicted model template and percent identity are provided in Table 3.

\section{Phylogeny analysis}

The phylogeny analysis was done using Mega7 software [53]. A best-fit model was selected using ProtTest [54] 3 which recommended $L G+G+F$, namely, the LG [55] amino acid substitution matrix, Gamma distribution (under four rate categories), and empirical amino acid frequencies, as the suitable model for the given alignment of UPOs and CPOs. A bootstrapped maximum likelihood (ML) tree was constructed with 1000 replicates using the recommended model. The LBA analysis was done using Tree puzzle 5.3 [56].

\section{Selection analyses}

The selection analysis was carried out on the Datamonkey web server [57] of HyPhy package [58]. Branch-specific and site-specific methods were applied to UPO and CPO sequences. Fast, Unconstrained Bayesian AppRoximation (FUBAR) [28], Mixed Effects Maximum Likelihood (MEME) [27],was used to identify pervasive and episodic selective pressure on each site respectively, and adaptive branch-site random effects likelihood (aBSREL) [30] was used as the branch-site model, and Branch-site unrestricted statistical test for episodic diversification (BUSTED) [26] was used to identify gene-wide test for positive selection on the entire phylogeny of UPOs and CPOs separately instead of selecting a few branches. This method tests for positive selection whether a gene has experienced at at least one site on at least one branch $\left(\omega_{1} \leq \omega_{2} \leq 1 \leq \omega_{3}\right)$ [26]. It applies the unconstrained model to estimate the proportion of sites per partition belonging to each $\omega$ class and then the constrained model by comparing the former model to a null model where $\omega_{3}=1$, which means it disallows positive selection on the foreground branches. These methods allow heterogeneous nonsynonymous $(\mathrm{dN})$ to 
Table 3 Summarizes the details of resultant UPO sequences showing their molecular weights and sequence identity with the template used to predict their structures

\begin{tabular}{|c|c|c|c|c|c|}
\hline $\begin{array}{l}\text { Peptide } \\
\text { sequence }\end{array}$ & Fungal species & $\begin{array}{l}\text { Number of Amino } \\
\text { acids }\end{array}$ & $\begin{array}{l}\text { Molecular weight } \\
(\mathrm{kDa})\end{array}$ & $\begin{array}{l}\text { Kind of UPO (based on structure } \\
\text { template) }\end{array}$ & $\begin{array}{l}\text { Sequence Identity with the } \\
\text { template (\%) }\end{array}$ \\
\hline PzperKZV89105 & Exidia glandulosa hhb12029 & 357 & 38.5 & AaeUPO & 58.62 \\
\hline EKM83318 & $\begin{array}{l}\text { Agaricus bisporus var burnettii } \\
\text { jb137s8 }\end{array}$ & 359 & 39.28 & AaeUPO & 65.74 \\
\hline GAC96564 & Pseudozyma hubeiensis sy62 & 242 & 26.45 & MroUPO & 27.52 \\
\hline KIM43689 & Hebeloma cylindrosporum h7 & 366 & 39.65 & AaeUPO & 62.93 \\
\hline KZS95533 & $\begin{array}{l}\text { Sistotremastrum niveocremeum } \\
\text { hhb9708 }\end{array}$ & 350 & 37.19 & AaeUPO & 41.81 \\
\hline KDQ50980 & Jaapia argillacea muc133604 & 323 & 35.14 & AaeUPO & 38.46 \\
\hline KIM85662 & Piloderma croceum f1598 & 358 & 39.89 & AaeUPO & 54.11 \\
\hline KZP09816 & Fibulorhizoctonia sp cbs 109695 & 366 & 39.19 & AaeUPO & 55.76 \\
\hline EJT50684 & $\begin{array}{l}\text { Trichosporon asahii var asahii } \\
\text { cbs } 2479\end{array}$ & 210 & 23.67 & MroUPO & 35.23 \\
\hline KIS69141 & Ustilago maydis & 265 & 29.24 & AaeUPO & 35.00 \\
\hline CCF52713 & Ustilago hordei & 244 & 27.13 & MroUPO & 26.15 \\
\hline KIJ40725 & Sphaerobolus stellatus ss 14 & 367 & 39.03 & AaeUPO & 66.56 \\
\hline EAU83038 & Coprinopsis cinerea okayama7.130 & 349 & 38.15 & AaeUPO & 63.58 \\
\hline EKC98656 & $\begin{array}{l}\text { Trichosporon asahii var asahii } \\
\text { cbss904 }\end{array}$ & 213 & 23.51 & MroUPO & 40.96 \\
\hline KDR72025 & Galerina marginata cbs 339.88 & 361 & 39.04 & AaeUPO & 71.52 \\
\hline KLT38585 & Cutaneotrichosporon oleaginosus & 216 & 24.06 & MroUPO & 29.35 \\
\hline KJA15764 & Hypholoma sublaterium fd334ss4 & 362 & 38.71 & AaeUPO & 63.84 \\
\hline CBQ73518 & Sporisorium reilianum & 241 & 26.46 & MroUPO & 27.06 \\
\hline EST07231 & Kalmanozyma brasiliensis ghg001 & 270 & 29.27 & AaeUPO & 36.54 \\
\hline EPE26857 & Glarea lozoyensis atcc20868 & 271 & 29.42 & AaeUPO & 31.22 \\
\hline KEQ59606 & $\begin{array}{l}\text { Aureobasidium melanogenum } \\
\text { cbs } 110374\end{array}$ & 227 & 24.25 & AaeUPO & 29.95 \\
\hline GAA97831 & Mixia osmundae iam14324 & 189 & 20.26 & AaeUPO & 33.33 \\
\hline KIK06072 & Laccaria amethystine laam08.1 & 366 & 39.61 & AaeUPO & 67.08 \\
\hline Mycgr3P74298 & Zymoseptoria tritici & 223 & 24.49 & MroUPO & 32.16 \\
\hline OAG12657 & Paraphaeosphaeria sporulosa & 219 & 23.54 & AaeUPO & 36.92 \\
\hline KPM41744 & Neonectria ditissima & 269 & 28.77 & AaeUPO & 33.91 \\
\hline KUJ12795 & Phialocephala scopiformis & 208 & 22.54 & AaeUPO & 34.31 \\
\hline OAJ19358 & Tilletia walkeri & 360 & 38.19 & AaeUPO & 56.78 \\
\hline KEQ68786 & $\begin{array}{l}\text { Aureobasidium namibiae } \\
\text { cbs } 147.97\end{array}$ & 214 & 22.53 & AaeUPO & 33.15 \\
\hline GAK67998 & Moesziomyces antarcticus & 245 & 26.74 & AaeUPO & 35.68 \\
\hline KXL42501 & Acidomyces richmondensis & 266 & 28.83 & AaeUPO & 33.61 \\
\hline OAJ12013 & Tilletia controversa & 359 & 38.48 & AaeUPO & 55.21 \\
\hline CZR67509 & Phialocephala subalpina & 208 & 22.72 & AaeUPO & 32.18 \\
\hline EMF09816 & Sphaerulina musiva so2202 & 250 & 27.11 & MroUPO & 31.92 \\
\hline KZT41007 & $\begin{array}{l}\text { Sistotremastrum suecicum } \\
\text { hhb10207ss3 }\end{array}$ & 292 & 31.71 & AaeUPO & 38.43 \\
\hline
\end{tabular}

synonymous $(\mathrm{dS})$ rate ratios $(\omega=\mathrm{dN} / \mathrm{dS})$ among the branches and across sites.

\section{Functional divergence analysis}

Type-I and Type-II functional divergence of the Basidiomycota and Ascomycota UPOs, along with CPOs was estimated in pairs using DIVERGE 3.0 [59] at a bootstrapping value of 100 . Type-I functional divergence coefficient $\left(\Theta_{\mathrm{I}}\right)$ identifies the sites having different evolutionary rates caused by the functional divergence [60] and Type-II functional divergence coefficient $\left(\Theta_{\text {II }}\right)$ identifies the radical amino acid changes at some sites 
caused by the rapid evolution [61]. Another Type-I Analysis [62] which provides site-specific profile and residues involved in functional divergence, was performed for the five non-degenerate patterns, S0 to S4, where S0 means no Type-I divergence in any cluster, S1 signifies Type-I divergence in cluster 1, and so on, finally S4 means all the clusters have experienced Type-I divergence.

\section{Additional files}

Additional file 1: Table S1 Number of putative sequences obtained in 35 different fungal species using the pipeline. (DOCX $13 \mathrm{~kb}$ )

Additional file 2: Figure S1 MSA of all UPO-encoding sequences and CPO sequences used as the outgroup. Red arrows point to PCP motif, green arrows point to EGD motif in UPOs, and blue arrows point to the acid-base catalyst pair in all UPOs. The asterisks $\left(^{*}\right)$ represent the conserved sites and \# represents the clipped gapped sites in the alignment. (TIF $68781 \mathrm{~kb}$ )

Additional file 3: Table S2 The binding cavity analysis of all the predicted structures of newly found UPOs. The binding pockets are shown in surface and the aromatic residues are shown in sticks. (DOCX $13720 \mathrm{~kb})$

Additional file 4: Figure S2 MSA of the five different subfamilies of UPOs and newly found motifs highlighted with rectangles: sky blue for Subfamily-I, pink for Subfamily-II, green for Subfamily-III, blue represents motifs in Subfamily-IV, yellow represents motifs in Subfamily-V and red for the motifs found in all UPOs. (TIF $27409 \mathrm{~kb}$ )

Additional file 5: Selection analyses data for UPOs. (XLSX $119 \mathrm{~kb}$ ) Additional file 6: Selection analyses data for CPOs. (XLSX $109 \mathrm{~kb}$ )

Additional file 7: Figure S3 MSA of all UPOs showing the positive and negatively selected sites using the MEME and FUBAR method. The asterisks $\left(^{*}\right)$ represent the conserved sites and the arrows point towards the motifs: PCP-EGD-R---E. (TIF $25856 \mathrm{~kb}$ )

Additional file 8: Figure S4 A graph showing the number of (a) synonymous and (b) nonsynonymous sites in UPOs obtained using the MEME method. (TIF $649 \mathrm{~kb}$ )

Additional file 9: Figure S5 Selection analysis on UPOs using aBSREL, a branch-site model. Thicker branches have a $p$-value $<0.05$ showing evidence of undergoing positive diversifying selection. (TIF $1225 \mathrm{~kb}$ )

Additional file 10: Figure S6 MSA of all CPOs showing the positive and negatively selected sites using the MEME and FUBAR method. The asterisks $\left(^{*}\right)$ represent the conserved sites and the arrows point towards the motifs: PCP-EHD---E. (TIF 17764 kb)

Additional file 11: Figure S7 A graph showing the number of (a) synonymous and (b) nonsynonymous sites in CPOs obtained using the MEME method. (TIF $602 \mathrm{~kb}$ )

Additional file 12: Figure $\mathbf{8 8}$ Selection analysis on CPOs using aBSREL, a branch-site model. Thicker branches have a p-value $<0.05$ showing evidence of positive diversifying selection. (TIF $1002 \mathrm{~kb}$ )

Additional file 13: Figure S9 An MSA of the clusters formed for the functional divergence analysis showing the Type-l functional divergent sites highlighted with black color. (TIF $7611 \mathrm{~kb}$ )

Additional file 14: Functional divergence analysis data for UPOs and CPOs. (XLSX $77 \mathrm{~kb})$

Additional file 15: Figure S10 Structural representation of the newly found motifs adhering near the binding pockets (shown as surface) are shown in one species from each subfamily of UPOs; a) experimentally resolved structure of AaeUPO and modeled structures of b) Mixia osmundae iam 14324, c) Jaapia argillacea mucl33604, d) Kalmanozyma brasiliensis ghg001, e) Glarea lozoyensis atcc20868, and f) Phialocephala scopiformis. (TIF $2498 \mathrm{~kb}$ )
Additional file 16: Table S3 Information of all the fungal genome sequences used in this study. (XLSX $38 \mathrm{~kb}$ )

Additional file 17: Table S4 CPO sequences and MroUPO used in this study. (DOCX $14 \mathrm{~kb}$ )

\section{Abbreviations}

Aae: Agrocybe aegerita; CPO: Chloroperoxidase; LBA: Long branch attraction; Lfu: Leptoxyphium fumago; Mro: Marasmius rotula; MSA: Multiple Sequence Alignment; Pog: Peroxidase-peroxygenase; UPO: Unspecific peroxygenase

\section{Acknowledgements}

Not applicable.

\section{Funding}

This work was supported by the National Outstanding Youth Science Foundation of China (31725022), Molecular Enzyme and Engineering International Cooperation Base of South China University of Technology (2017A050503001), Special Program of Guangdong Province for Leader Project in Science and Technology Innovation: Development of New Partial Glycerin Lipase (2015TX01N207), Science and Technology Planning Project of Guangdong Province (2016B090920082), National Supercomputer Center in Guangzhou and the Special Program for Applied Research on Super Computation of the NSFC-Guangdong Joint Fund, and Marine S\&T Fund of Shandong Province (2018SDKJ0302-2). Funders had no role in the design of the study and collection, analysis, and interpretation of data and in writing the manuscript.

\section{Availability of data and materials}

The datasets supporting the conclusions of this study are included within the article (and its Additional files).

\section{Authors' contributions}

The original idea of this study was conceived by YW, SH, and DL. MF and SH designed the experiments performed by MF and collected the data. All authors analyzed the data. The manuscript was drafted by MF and SH critically revised by all the co-authors. All authors read and approved the final manuscript.

Ethics approval and consent to participate

Not applicable.

\section{Consent for publication}

Not applicable.

\section{Competing interests}

The authors declare that they have no competing interests.

\section{Publisher's Note}

Springer Nature remains neutral with regard to jurisdictional claims in published maps and institutional affiliations.

\section{Author details}

${ }^{1}$ School of Food Science and Engineering, South China University of Technology, Guangzhou 510640, China. ${ }^{2}$ Laboratory for Marine Biology and Biotechnology, Qingdao National Laboratory for Marine Science and Technology, Qingdao, China. ${ }^{3}$ State Key Laboratory of Biocontrol, School of Life Sciences, Sun Yat-Sen University, Guangzhou, China.

Received: 22 October 2018 Accepted: 20 February 2019 Published online: 13 March 2019

\section{References}

1. Ullrich R, Nüske J, Scheibner K, Spantzel J, Hofrichter M. Novel haloperoxidase from the agaric basidiomycete Agrocybe aegerita oxidizes aryl alcohols and aldehydes. Appl Environ Microbiol [Internet]. 2004;70(8): 4575-81. Available from: http://www.ncbi.nlm.nih.gov/pubmed/15294788. [cited 2018 Jun 16].

2. Index Fungorum [Internet]. 2013. Available from: www.indexfungorum.org 
3. Stamets $P$, Chilton J. The mushroom cultivator: a pratical guide to growing mushrooms at home [Internet]. Agarikon Press, Olympia; 1983 [cited 2018 Jun 17]. Available from: http://agris.fao.org/agris-search/search.do?recordID= XF2015041055

4. Peter S, Kinne M, Wang X, Ullrich R, Kayser G, Groves JT, et al. Selective hydroxylation of alkanes by an extracellular fungal peroxygenase. FEBS J. 2011;278(19):3667-75 Available from: http://doi.wiley.com/10.1111/j.17424658.2011.08285.x. [cited 2018 Jun 17].

5. Gutiérrez A, Babot ED, Ullrich R, Hofrichter M, Martínez AT, del Río JC. Regioselective oxygenation of fatty acids, fatty alcohols and other aliphatic compounds by a basidiomycete heme-thiolate peroxidase. Arch Biochem Biophys. 2011;514(1-2):33-43 Available from: http://linkinghub.elsevier.com/ retrieve/pii/S000398611100289X. [cited 2018 Jun 17].

6. Hofrichter M, Ullrich R. Oxidations catalyzed by fungal peroxygenases. Curr Opin Chem Biol. 2014;19:116-25 Available from: https://www.sciencedirect. com/science/article/pii/S1367593114000106. [cited 2018 Jun 16].

7. Hofrichter M, Kellner H, Pecyna MJ, Ullrich R. Fungal Unspecific Peroxygenases: Heme-Thiolate Proteins That Combine Peroxidase and Cytochrome P450 Properties. In: Advances in experimental medicine and biology [Internet]; 2015. [cited 2018 Jun 19]. p. 341-68. Available from: http://www.ncbi.nlm.nih.gov/pubmed/26002742.

8. Wang $Y$, Lan D, Durrani R, Hollmann F. Peroxygenases en route to becoming dream catalysts. What are the opportunities and challenges? Curr Opin Chem Biol. 2017;37:1-9.

9. Bordeaux M, Galarneau A, Drone J. Catalytic, Mild, and Selective Oxyfunctionalization of Linear Alkanes: Current Challenges. Angew Chemie Int Ed. 2012;51(43):10712-23 Available from: http://www.ncbi.nlm.nih.gov/ pubmed/22996726. [cited 2018 Jul 27].

10. Hofrichter M, Ullrich R. Heme-thiolate haloperoxidases: versatile biocatalysts with biotechnological and environmental significance. Appl Microbiol Biotechnol. 2006;71(3):276-88 Available from: http://link.springer.com/10 1007/s00253-006-0417-3. [cited 2018 Jun 17].

11. Hofrichter M, Ullrich R, Pecyna MJ, Liers C, Lundell T. New and classic families of secreted fungal heme peroxidases. Appl Microbiol Biotechnol. 2010;87(3):871-97 Available from: http://link.springer.com/10.1007/s00253010-2633-0. [cited 2018 Jun 17].

12. Ruiz-Dueñas FJ, Martínez AT. Structural and Functional Features of Peroxidases with a Potential as Industrial Biocatalysts. In: Biocatalysis Based on Heme Peroxidases [Internet]. Berlin: Springer Berlin Heidelberg; 2010. p. 37-59. Available from: http://link.springer.com/10.1007/978-3-642-12627-7_3. [cited 2018 Jun 17].

13. Kellner H, Luis P, Pecyna MJ, Barbi F, Kapturska D, Krüger D, et al. Widespread Occurrence of Expressed Fungal Secretory Peroxidases in Forest Soils. PLoS One. 2014;9(4):e95557 Available from: http://dx.plos.org/10.1371/ journal.pone.0095557. [cited 2018 Jun 18].

14. Piontek K, Strittmatter E, Ullrich R, Gröbe G, Pecyna MJ, Kluge M, et al. Structural basis of substrate conversion in a new aromatic peroxygenase: cytochrome P450 functionality with benefits. J Biol Chem. 2013;288(48):34767-76.

15. Gröbe G, Ullrich R, Pecyna MJ, Kapturska D, Friedrich S, Hofrichter M, et al. Highyield production of aromatic peroxygenase by the agaric fungus Marasmius rotula. AMB Express [Internet]. 2011;1(1):1-11. Available from: http://amb-express. springeropen.com/articles/10.1186/2191-0855-1-31. [cited 2018 Jul 28].

16. Poraj-Kobielska M, Kinne M, Ullrich R, Scheibner K, Kayser G, Hammel KE, et al. Preparation of human drug metabolites using fungal peroxygenases. Biochem Pharmacol [Internet]. 2011;82(7):789-96. Available from: https:// www.sciencedirect.com/science/article/abs/pii/S0006295211004035. [cited 2018 Jul 14]

17. Anh DH, Ullrich R, Benndorf D, Svatos A, Muck A, Hofrichter M. The Coprophilous Mushroom Coprinus radians Secretes a Haloperoxidase That Catalyzes Aromatic Peroxygenation. Appl Environ Microbiol. 2007;73(17): 5477-85 Available from: http://www.ncbi.nlm.nih.gov/pubmed/17601809. [cited 2018 Dec 6]

18. Baciocchi E, Fabbrini M, Lanzalunga O, Manduchi L, Pochetti G. Prochiral selectivity in $\mathrm{H} 2 \mathrm{O} 2$-promoted oxidation of arylalkanols catalysed by chloroperoxidase: The role of the interactions between the $\mathrm{OH}$ group and the amino-acid residues in the enzyme active site. Eur J Biochem. 2001; 268(3):665-72 Available from: http://doi.wiley.com/10.1046/j.1432-1327.2001. 01924.x. [cited 2018 Jul 28].

19. Poulos TL, Kraut J. The stereochemistry of peroxidase catalysis. Journal of Biological Chemistry. 1980;255 Available from: http://www.jbc.org/content/ 255/17/8199.full.pdf. [cited 2018 Jul 30].
20. Strittmatter E, Liers C, Ullrich R, Wachter S, Hofrichter M, Plattner DA, et al. First crystal structure of a fungal high-redox potential dye-decolorizing peroxidase substrate interaction sites and long-range electron transfer. J Biol Chem. 2013;288(6):4095-102 Available from: http://www.ncbi.nlm.nih.gov/ pubmed/23235158. [cited 2018 Jul 30].

21. Sundaramoorthy $M$, Terner J, Poulos TL. The crystal structure of chloroperoxidase: a heme peroxidase--cytochrome P450 functional hybrid. Structure. 1995;3(12):1367-77 Available from: http://www.ncbi.nlm.nih.gov/ pubmed/8747463.

22. Pecyna MJ, Ullrich R, Bittner B, Clemens A, Scheibner K, Schubert R, et al. Molecular characterization of aromatic peroxygenase from Agrocybe aegerita. Appl Microbiol Biotechnol. 2009;84(5):885-97.

23. Strimmer K, von Haeseler A. Likelihood-mapping: a simple method to visualize phylogenetic content of a sequence alignment. Proc Natl Acad Sci U S A. 1997;94(13):6815-9 Available from: http://www.ncbi.nlm.nih.gov/ pubmed/9192648. [cited 2018 Jun 20].

24. Morin E, Kohler A, Baker AR, Foulongne-Oriol M, Lombard V, Nagye LG, et al. Genome sequence of the button mushroom Agaricus bisporus reveals mechanisms governing adaptation to a humic-rich ecological niche. Proc Natl Acad Sci. 2012;109(43):17501-6 Available from: http://www.pnas.org/ cgi/doi/10.1073/pnas.1206847109. [cited 2018 Jul 9].

25. Tuynman A, Spelberg JL, Kooter IM, Schoemaker HE, Wever R. Enantioselective epoxidation and carbon-carbon bond cleavage catalyzed by Coprinus cinereus peroxidase and myeloperoxidase. J Biol Chem. 2000; 275(5):3025-30 Available from: http://www.ncbi.nlm.nih.gov/pubmed/ 10652281. [cited 2018 Oct 9].

26. Murrell B, Weaver S, Smith MD, Wertheim JO, Murrell S, Aylward A, et al. Gene-Wide Identification of Episodic Selection. Mol Biol Evol. 2015;32(5): 1365-71 Available from: https://academic.oup.com/mbe/article-lookup/doi/ 10.1093/molbev/msv035. [cited 2018 Jul 13].

27. Murrell B, Wertheim JO, Moola S, Weighill T, Scheffler K, Kosakovsky Pond SL. Detecting Individual Sites Subject to Episodic Diversifying Selection. Malik HS, editor. PLoS Genet [Internet]. 2012;8(7):e1002764. Available from: https://dx.plos.org/10.1371/journal.pgen.1002764. [cited 2018 Dec 25].

28. Murrell B, Moola S, Mabona A, Weighill T, Sheward D, Kosakovsky Pond SL, et al. FUBAR: A Fast, Unconstrained Bayesian AppRoximation for Inferring Selection. Mol Biol Evol [Internet]. 2013;30(5):1196-205. Available from: https://academic.oup.com/mbe/article-lookup/doi/10.1093/molbev/mst030. [cited 2018 Dec 2].

29. Loewe L. Negative Selection. Nat Educ. 2008;59(1). Available from: https:// www.nature.com/scitable/topicpage/negative-selection-1136

30. Smith MD, Wertheim JO, Weaver S, Murrell B, Scheffler K, Kosakovsky Pond SL. Less Is More: An Adaptive Branch-Site Random Effects Model for Efficient Detection of Episodic Diversifying Selection. Mol Biol Evol. 2015;32(5):134253 Available from: https://academic.oup.com/mbe/article-lookup/doi/10. 1093/molbev/msv022.

31. Sundaramoorthy M, Terner J, Poulos TL. The crystal structure of chloroperoxidase: a heme peroxidase-cytochrome P450 functional hybrid. Structure. 1995;3(12):1367-78.

32. Kedderis GL, Hollenberg PF. Steady state kinetics of chloroperoxidase-catalyzed N-demethylation reactions. J Biol Chem. 1983;258(20):12413-9 Available from: http://www.jbc.org/content/258/20/12413.short. [cited 2018 Sep 12].

33. Cooke LR, Watters BS, Brown AE. The effect of fungicide sprays on the incidence of apple canker (Nectria galligena) in Bramley's Seedling. Plant Pathol. 1993;42(3):432-42 Available from: http://doi.wiley.com/10.1111/j. 1365-3059.1993.tb01522.x

34. Stukenbrock EH, Jørgensen FG, Zala M, Hansen TT, BA MD, Schierup MH. Whole-Genome and Chromosome Evolution Associated with Host Adaptation and Speciation of the Wheat Pathogen Mycosphaerella graminicola. PLoS Genet. 2010;6(12):e1001189 Available from: http://dx.plos. org/10.1371/journal.pgen.1001189. [cited 2018 Jul 2].

35. Quaedvlieg W, GJM V, Shin H-D, Barreto RW, Alfenas AC, Swart WJ, et al. Sizing up Septoria. Stud Mycol. 2013;75(1):307-90 Available from: http:// www.ncbi.nlm.nih.gov/pubmed/24014902. [cited 2018 Jul 2].

36. Gostinčar C, Ohm RA, Kogej T, Sonjak S, Turk M, Zajc J, et al. Genome sequencing of four Aureobasidium pullulans varieties: biotechnological potential, stress tolerance, and description of new species. BMC Genomics. 2014;15(1):549 Available from: http://www.ncbi.n/m.nih.gov/pubmed/ 24984952. [cited 2018 Jul 2].

37. Mosier AC, Miller CS, Frischkorn KR, Ohm RA, Li Z, LaButti K, et al. Fungi Contribute Critical but Spatially Varying Roles in Nitrogen and Carbon 
Cycling in Acid Mine Drainage. Front Microbiol. 2016;7:238 Available from: http://www.ncbi.nlm.nih.gov/pubmed/26973616. [cited 2018 Jul 2].

38. Reininger V, Schlegel M. Analysis of the Phialocephala subalpina Transcriptome during Colonization of Its Host Plant Picea abies. PLoS One. 2016;11(3):e0150591 Available from: http://dx.plos.org/10.1371/journal.pone. 0150591. [cited $2018 \mathrm{Jul} 2$ ].

39. Banuett F. Genetics of Ustilago maydis, a Fungal Pathogen that Induces Tumors in Maize. Annu Rev Genet. 1995;29(1):179-208 Available from: http://www.ncbi.nlm.nih.gov/pubmed/8825473. [cited 2018 Jul 2].

40. Schirawski J, Mannhaupt G, Munch K, Brefort T, Schipper K, Doehlemann G, et al. Pathogenicity Determinants in Smut Fungi Revealed by Genome Comparison. Science. 2010;330(6010):1546-8 Available from: http://www. ncbi.nlm.nih.gov/pubmed/21148393. [cited 2018 Jul 2].

41. Chan GF, Bamadhaj HM, Gan HM, Aini N, Rashid A, Malaysia T, et al. Genome Sequence of Aureobasidium pullulans AY4, an Emerging Opportunistic Fungal Pathogen with Diverse Biotechnological Potential. 2012; Available from:https://ec.asm.org/content/11/11/1419.short.

42. Chen L, Yue Q, Zhang X, Xiang M, Wang C, Li S, et al. Genomics-driven discovery of the pneumocandin biosynthetic gene cluster in the fungus Glarea lozoyensis. BMC Genomics. 2013;14(1):339 Available from: http:// www.ncbi.nlm.nih.gov/pubmed/23688303. [cited 2018 Jul 8].

43. Balkovec JM, Hughes DL, Masurekar PS, Sable CA, Schwartz RE, Singh SB. Discovery and development of first in class antifungal caspofungin (CANCIDAS ${ }^{\odot}$-A case study. Nat Prod Rep. 2014;31(1):15-34 Available from: http://Xlink.rsc.org/?DOl=C3NP70070D. [cited 2018 Jul 8].

44. Betts MJ, Russell RB. Amino acid properties and consequences of substitutions. In: Gray IC, editor. Bioinformatics for Geneticists [Internet]: Wiley; 2003. Available from: http://www.russelllab.org/aas/lle.html. [cited 2018 Jul 13].

45. Morgan CC, Shakya K, Webb A, Walsh TA, Lynch M, Loscher CE, et al. Colon cancer associated genes exhibit signatures of positive selection at functionally significant positions. BMC Evol Biol. 2012;12(1):114 Available from: http://bmcevolbiol.biomedcentral.com/articles/10.1186/1471-2148-12114. [cited 2018 Sep 9].

46. Kersey PJ, Allen JE, Allot A, Barba M, Boddu S, Bolt BJ, et al. Ensembl Genomes 2018: an integrated omics infrastructure for non-vertebrate species. Nucleic Acids Res. 2018;46(D1):D802-8 Available from: http:// academic.oup.com/nar/article/46/D1/D802/4577569. [cited 2018 Jul 10].

47. NCBI [Internet]. Available from: www.ncbi.nlm.nih.gov

48. Li W, Godzik A. Cd-hit: a fast program for clustering and comparing large sets of protein or nucleotide sequences. Bioinformatics. 2006;22(13):1658-9 Available from: http://www.ncbi.nlm.nih.gov/pubmed/16731699. [cited 2018 Jun 19].

49. Dongen S. A cluster algorithm for graphs; 2000. Available from: https://dl. acm.org/citation. $\mathrm{fm}$ ?id=868986.

50. Jeanmougin F, Thompson JD, Gouy M, Higgins DG, Gibson TJ. Multiple sequence alignment with Clustal X. Trends Biochem Sci. 1998;23:403-5 Available from: https://hal.inria.fr/hal-00428491/. [cited 2017 Apr 22].

51. Waterhouse A, Bertoni M, Bienert S, Studer G, Tauriello G, Gumienny R, et al. SWISS-MODEL: homology modelling of protein structures and complexes. Nucleic Acids Res. 2018; Available from: https://academic.oup.com/nar/ advance-article/doi/10.1093/nar/gky427/5000024. [cited 2018 Jun 19].

52. Schrodinger LL. The PyMOL molecular graphics system. Version. 2010;1(5):0.

53. Kumar S, Stecher G, Tamura K. MEGA7: Molecular Evolutionary Genetics Analysis Version 7.0 for Bigger Datasets. Mol Biol Evol. 2015;33(7):1870-4 Available from: https://www.megasoftware.net/pdfs/KumarStecher16.pdf. [cited 2018 Jun 19].

54. Darriba D, Taboada GL, Doallo R, Posada D. ProtTest 3: fast selection of bestfit models of protein evolution. Bioinformatics. 2011;27(8):1164-5 Available from: https://academic.oup.com/bioinformatics/article-lookup/doi/10.1093/ bioinformatics/btr088. [cited 2018 Dec 11].

55. Le SQ, Gascuel O. An Improved General Amino Acid Replacement Matrix. Mol Biol Evol. 2008;25(7):1307-20 Available from: https://academic.oup.com/ mbe/article-lookup/doi/10.1093/molbev/msn067. [cited 2018 Dec 11].

56. Schmidt HA, Strimmer K, Vingron M, von Haeseler A. TREE-PUZZLE: maximum likelihood phylogenetic analysis using quartets and parallel computing. Bioinformatics. 2002;18(3):502-4 Available from: https:// academic.oup.com/bioinformatics/article-lookup/doi/10.1093/bioinformatics/ 18.3.502. [cited 2018 Jun 20].

57. Weaver S, Shank SD, Spielman SJ, Li M, Muse SV, Kosakovsky Pond SL. Datamonkey 2.0: A Modern Web Application for Characterizing Selective and Other Evolutionary Processes. Mol Biol Evol. 2018;35(3):773-7 Available from: https:/academic.oup.com/mbe/article/35/3/773/4782511. [cited 2018 Jul 13].

58. Pond SLK, Frost SDW, Muse S V. HyPhy: hypothesis testing using phylogenies. Bioinformatics [Internet]. 2005;21(5):676-9. Available from: https://academic.oup.com/bioinformatics/article-lookup/doi/10.1093/ bioinformatics/bti079. [cited $2018 \mathrm{Jul} 13]$.

59. Gu X. DIVERGE MANUAL version 3.0 ( DetectIng Variability in Evolutionary Rates among GEnes). 2013;0:0-50.

60. Gu X, Vander Velden K. DIVERGE: phylogeny-based analysis for functionalstructural divergence of a protein family. Bioinformatics. 2002;18(3):500-1 Available from: https:/academic.oup.com/bioinformatics/article-lookup/doi/ 10.1093/bioinformatics/18.3.500.

61. Gu X. A Simple Statistical Method for Estimating Type-II (Cluster-Specific) Functional Divergence of Protein Sequences. Mol Biol Evol. 2006;23(10):193745 Available from: http://academic.oup.com/mbe/article/23/10/1937/1096967/ A-Simple-Statistical-Method-for-Estimating-Typell. [cited 2018 Aug 20].

62. Gu X. A Site-specific Measure for Rate Difference After Gene Duplication or Speciation. Mol Biol Evol. 2001;18(12):2327-30 Available from: http:// academic.oup.com/mbe/article/18/12/2327/1074383. [cited 2018 Sep 13].
Ready to submit your research? Choose BMC and benefit from:

- fast, convenient online submission

- thorough peer review by experienced researchers in your field

- rapid publication on acceptance

- support for research data, including large and complex data types

- gold Open Access which fosters wider collaboration and increased citations

- maximum visibility for your research: over $100 \mathrm{M}$ website views per year

At $\mathrm{BMC}$, research is always in progress.

Learn more biomedcentral.com/submissions 